\title{
Fast X-Ray Diffraction (XRD) Tomography for Enhanced Identification of Materials
}

This paper was downloaded from TechRxiv (https://www.techrxiv.org).

\section{LICENSE}

CC BY-NC-SA 4.0

SUBMISSION DATE / POSTED DATE

$04-12-2021 / 10-12-2021$

\section{CITATION}

Korolkovas, Airidas; Katsevich, Alexander; Frenkel, Michael; Thompson, William; Morton, Edward (2021): Fast X-Ray Diffraction (XRD) Tomography for Enhanced Identification of Materials. TechRxiv. Preprint. https://doi.org/10.36227/techrxiv.17125448.v1

DOI 


\title{
Fast X-Ray Diffraction (XRD) Tomography for Enhanced Identification of Materials
}

\author{
Airidas Korolkovas, Alexander Katsevich, Michael Frenkel, William Thompson, Edward Morton
}

\begin{abstract}
X-ray computed tomography (CT) can provide 3D images of density, and possibly the atomic number, for large objects like passenger luggage. This information, while generally very useful, is often insufficient to identify threats like explosives and narcotics, which can have a similar average composition as benign everyday materials such as plastics, glass, light metals, etc. A much more specific material signature can be measured with X-ray diffraction (XRD). Unfortunately, XRD signal is very faint compared to the transmitted one, and also challenging to reconstruct for objects larger than a small laboratory sample. In this article we analyze a novel low-cost scanner design which captures CT and XRD signals simultaneously, and uses the least possible collimation to maximize the flux. To simulate a realistic instrument, we derive a formula for the resolution of any diffraction pathway, taking into account the polychromatic spectrum, and the finite size of the source, detector, and each voxel. We then show how to reconstruct XRD patterns from a large phantom with multiple diffracting objects. Our approach includes a reasonable amount of photon counting noise (Poisson statistics), as well as measurement bias, in particular incoherent Compton scattering. The resolution of our reconstruction is sufficient to provide significantly more information than standard CT, thus increasing the accuracy of threat detection. Our theoretical model is implemented in GPU (Graphics Processing Unit) accelerated software which can be used to assess and further optimize scanner designs for specific applications in security, healthcare, and manufacturing quality control.
\end{abstract}

\section{Index Terms}

X-ray diffraction, tomography, maximum likelihood detection, maximum likelihood estimation, signal reconstruction, reconstruction algorithms, parallel algorithms, air safety, hazardous materials.

\section{INTRODUCTION}

\section{A. Literature overview}

$\mathbf{X}$ RAY diffraction (XRD) is a well-established modality to probe material structure at the molecular level. It is especially well-suited for studying crystals, since they have highly periodic structures which give rise to sharp, distinct diffraction peaks. This property is useful in airport security screenings to detect illicit and hazardous substances, like narcotics and explosives, since many of them have a substantial amount of crystallinity. On the flip side, harmless metals, ceramics, and plastics may diffract X-rays equally or even more strongly. Fortunately, the diffraction pattern of each material is quite unique, and can be fingerprinted if we have an extensive database of previously measured materials that can possibly be found in passenger baggage.

While XRD is most sensitive to crystalline materials, it can be used to detect amorphous and some liquid ones as well. In Ref. [1] the authors have reconstructed diffraction patterns of a phantom containing water, fat, collagen, and tricalcium phosphate (a substitute for bone). These biological materials are not crystalline, hence their diffraction patterns are quite smooth. Nevertheless, XRD reconstruction was able to clearly distinguish between them.

The fidelity of XRD-based material identification depends on the resolution at which we are able to extract its diffraction pattern. An example of a good resolution obtained in a real airport security setting is described in Ref. [2]. Their workflow consists of two machines: first a CT (computed tomography) scan to identify potential threat objects, and then a second pass through an X-ray diffractometer to provide a more specific material signature. To measure XRD at high resolution, the authors have restricted the X-ray beam aperture to a thin pencil shape, as well as added collimators in front of their detector so that it accepts only a narrow range of scattering angles. Using an X-ray tube operating at $1.6 \mathrm{~kW}$, the authors have scanned 4182 passenger baggage articles over the course of 53 days, or 3-4 items per hour. Despite these promising results, further improvements in speed and accuracy are needed before this system can be widely deployed in commercial aviation.

Because of their minuscule refraction coefficient, X-rays cannot be optically collimated within reasonable distances of $\mathcal{O}(1 \mathrm{~m})$ that are available in typical security or medical screening rooms. Synchrotron sources are an exception, since they

This draft was last modified on 12/02/2021. This research was funded in part by the U.S. Department of Homeland Security, Science and Technology Directorate under a competitively awarded contract: 70-RSAT-18-C-B0000047. This support does not constitute an express or implied endorsement on the part of the Government.

Airidas Korolkovas and Michael Frenkel are with iTomography Corporation, Houston, TX 77021, USA. (e-mail: airidas@itomography.com, michael@itomography.com)

Alexander Katsevich is with the University of Central Florida, Orlando, FL 32816, USA, and with iTomography Corporation, Houston, TX 77021, USA. (e-mail: alexander.katsevich@ucf.edu and alexander@itomography.com)

William Thompson and Edward Morton are with Rapiscan Systems, 2805 Columbia St, Torrance, CA 90503, USA (e-mail: emorton@rapiscansystems.com, withompson@rapiscansystems.com) 
produce intrinsically collimated X-ray beams, which can be further focused with $\mathcal{O}(10 \mathrm{~m})$-long optics under high vacuum. XRD microtomography is routinely performed using dedicated synchrotron beamlines, to reveal spatially resolved diffraction patterns of various engineering materials, for example concrete, see Ref. [3]. While their data is of excellent quality, its acquisition has lasted 8 hours, which is too slow for being viable in airport security, healthcare, or manufacturing.

Since true collimation is not practical, radiation from X-ray tubes is usually delimited by placing shielding to create the desired beam shape. A major drawback of delimitation is the loss of X-ray flux, and a consequent increase in scan time. However, reasonable XRD tomography speeds have been demonstrated even with thin pencil-beam sources, especially if they use the full polychromatic spectrum, and a fairly open coded aperture in front of the detector, see Ref. [4]. In Ref. [5] the authors have even used a monochromatic pencil-beam, but the loss of flux was offset by combining CT and XRD reconstructions into a single edge-preserving algorithm, and forcing the diffraction patterns to be constant for each object in the phantom. In a subsequent study, Ref. [6], the same authors have used a coded aperture instead of detector collimators, which has increased the flux, but decreased the resolution. To maintain acceptable resolution, the authors have emphasized the importance of image segmentation, as well as using multi-energy CT information to better account for attenuation along the diffracted beam pathways.

In the case of very low photon counts that are required for medical breast imaging, Ref. [7] has demonstrated that Poisson likelihood maximization gives superior XRD reconstruction quality, compared to filtered back projection (FBP), whose main advantage in turn is computational speed.

Continuing improvements in X-ray tube technologies [8], as well as the increasing availability of pixelated and energyresolving detectors [9], have enabled novel and more affordable XRD imaging modalities. For explosives detection, Ref. [10] gives a detailed introduction on coherent scatter computed tomography, energy-dispersive X-ray diffraction tomography, as well as Compton back-scatter imaging. When discussing future prospects, Ref. [11] points to multi-focus X-ray sources (MFXS), where a single electron beam is deflected to multiple anodes, thus avoiding the need for moving parts, which should help with increasing scan speeds and decreasing maintenance costs. Ref. [12] showcases the importance of detector energy resolution. They have characterized their CdTe detector and found that its relative energy sensitivity is about $6 \%$ at momentum transfer of $2.5 \mathrm{~nm}^{-1}$.

One way to increase the flux is to use as many emitted photons as possible. In XRD tomography one can replace the pencil beam with a more luminous fan beam, in conjunction with detectors collimated perpendicular to the fan plane, see Ref. [13]. This geometry is sometimes called a "third generation XRD-CT", and has been verified experimentally in Ref. [14]. Reconstruction of one slice results in a $(1+2)$-dimensional image, i.e. the one-dimensional diffraction pattern is resolved in a two-dimensional plane. This system has been patented, see Ref. [15].

In Ref. [16] the authors have simultaneously measured both CT and XRD signals, using a polychromatic fan beam. The energy-resolving detector was collimated to increase the resolution, at the expense of flux. The reconstructed XRD patterns have a reasonably high quality, although the paper does not mention the measurement time needed.

An even greater flux can be achieved by replacing detector collimators with a coded aperture, Ref. [17]. This study has only used two views per scan (snapshot tomography) and has imaged a single water vial. Alternatively, imaging time can be reduced by a factor of 6 , if diffraction only needs to be reconstructed from a small region of the illuminated slice, see Ref. [18].

Generally, adding more collimators will increase the resolution and reduce the computational complexity of image reconstruction, at the expense of lower flux and/or longer measurement time [19]. Computational cost can also be reduced by using filtered back projection (FBP), which in the case of XRD tomography in $1+2$ dimensions has been shown to improve the reconstruction speed by two orders of magnitude [20].

So far, none of the XRD imaging designs has been commercialized and successfully deployed in real-world security settings. One of the main obstacles is imaging time, which consists of data acquisition and computational reconstruction, which in total is not much faster than hand-searching the luggage. To offset the slow speed, XRD imaging should be cheap, at least comparable to the cost of manual search labor. Lastly, while neither speed nor cost may be particularly impressive, XRD imaging has a unique advantage, in that it can identify various materials with a much higher specificity than any other modalities currently in use. Overall, there is a tradeoff between the resolution, speed, and cost of the system. Our aim in this theoretical paper is to strike a reasonable balance between these competing goals and demonstrate how the overall design could be valuable in practice.

\section{B. Outline of this article}

In this article, we present a design of an XRD tomographic imaging system that can be adapted for a variety of security applications. A key requirement is to decrease the XRD scan time to be on par with the already widespread CT modality, while keeping any new hardware costs to a minimum. Due to these practical constraints, the resolution of XRD data will be limited, but combined imaging will still be more informative than CT alone. With multi-energy imaging, CT can at most provide two numbers on each material, for example the density and the effective atomic number. If the XRD modality is capable to deliver at least one additional parameter per material, it would already lead to a significant improvement of threat detection capability. Here we are not concerned about the computational speed, as the price of computing hardware like GPUs (Graphics Processing 
Units) and cloud services continues to decrease, and the performance of GPUs improves steadily, whereas the price of X-ray imaging components like sources and detectors is relatively fixed. In other words, we intend to rely heavily on computation, instead of expensive high-quality imaging hardware.

Like many previous studies, we shall use a polychromatic fan-beam and a $2 \mathrm{D}$ energy-resolving detector. A key novelty is that our detector will not have any delimitators, be it collimators or a coded aperture. To the best of our knowledge, this results in the highest achievable flux for XRD tomography. The lack of collimation does not hinder XRD reconstruction, as long as the phantom can be segmented into a handful of objects, each having a uniform material composition. A similar idea was implemented in Ref. [6], except that we remove the coded aperture, use a larger number of views, and install more detector pixels.

Our imaging workflow relies heavily on combining CT and XRD modalities. We utilize just one illumination for both CT and XRD images, hence the only upgrade to an existing CT scanner is more detectors. The operational cost of the XRD add-on is minimal, since it takes the same time and almost the same power to record only CT, or both CT and XRD signals. We reconstruct the CT image first, which we then decompose into distinct objects of known attenuation. Only if the CT image shows a potential threat object of a substantial size, a tentative alarm is triggered and a secondary XRD reconstruction is launched. The main contribution of this article is a detailed model of the XRD image formation, assuming that all relevant scanner parameters and the transmission CT image are known. Our model is a linear system of $\mathcal{O}\left(10^{6}\right)$ equations (one for each relevant source-detector-energy bin) and $\mathcal{O}\left(10^{3}\right)$ unknowns, which are the handful of one-dimensional diffraction patterns. At face value this looks like a highly overdetermined system, hence it should be easy to solve. However, as we shall see, the information contained in each equation is strongly overlapping. This redundancy stems from the intrinsically low resolution which is due to poor collimation, as well as the source and the detector physics limitations of presently available hardware. Furthermore, the photon counts can be very low, resulting in many measurements with zero counts (the output is sparse). Lastly, the diffraction patterns are not free to assume just any random shape, so out of the $\mathcal{O}\left(10^{3}\right)$ unknowns perhaps just $\mathcal{O}(10)$ are truly independent. To sum up, we will be reconstructing a dozen of degrees of freedom from a million measurements, while fully anticipating that those measurements will have poor resolution and high noise.

\section{METHOD}

A diffraction scan data consists of $M=\mathrm{SRC} * \mathrm{COL} * \mathrm{ROW} * \mathrm{NRG}$ measurements. In this paper we will show one example with $\mathrm{SRC}=32$ source positions (view angles), $\mathrm{COL}=1024$ detector columns, $\mathrm{ROW}=1$ detector rows, and $\mathrm{NRG}=64$ energy channels. The unknowns will be $K=\mathrm{NWT} *$ MAT, where NWT $=256$ is the number of wavevector transfer grid points, and MAT $=3$ is the number of materials in the phantom. As we shall see, the number of photons $\mathbf{N}=N(m)$ detected at each $m=1,2, \ldots, M$ measurement can be expressed in standard linear algebra form (matrix multiplication with a column vector):

$$
\mathbf{N}=\mathbb{A} \cdot \mathbf{F}
$$

Here $\mathbf{F}=F(k)$ is a column vector containing all $k=1,2, \ldots, K$ unknowns. The model matrix $\mathbb{A}$ has the size $[M, K]$ and the main contribution of this work is showing how to obtain all the elements of the matrix. It is a computationally challenging task that we have implemented on GPU using CUDA (Compute Unified Device Architecture). A typical time for building the whole matrix with currently available GPU cards is approximately 1 hour, but can vary a lot depending on the size and resolution of the phantom and the spectrum. Further speedup, via better software and multi-GPU hardware, is possible. Once the matrix $\mathbb{A}$ is known, a full arsenal of linear algebra solvers can be used to find the inverse $\mathbf{F}=\mathbb{A}^{-1} \cdot \mathbf{N}$, which reveals the diffraction patterns $\mathbf{F}$, given the photon counts $\mathbf{N}$.

We begin by computing the expected number of single-diffracted photons at measurement $m$. It is a sum over all the (discretized) scattering pathways, each of which can be broken down into four factors:

1) geometrical factor,

2) source spectrum and detector response to it,

3) attenuation factor, given by the photon survival probability from the source to the voxel and from the voxel to the detector,

4) differential scattering cross-section per unit volume of material (depends on the energy and scattering angle)

In brief mathematical form, the number of scattered photons can be written as:

$$
N(m)=\sum_{\text {vox }=1}^{\text {vOX }} \sum_{\text {nrgsrc }=1}^{\text {NRG }}(G=\text { geometry })(\eta=\text { spectrum })\left(P_{\text {in }} P_{\text {out }}=\text { attenuation }\right)(F=\text { cross-section })
$$

In the following sections we explain each factor in detail and show how to compute it. In the end, we will factor out the unknown $F(k)$ from the sum, and what remains will be the matrix $\mathbb{A}(m, k)$. 


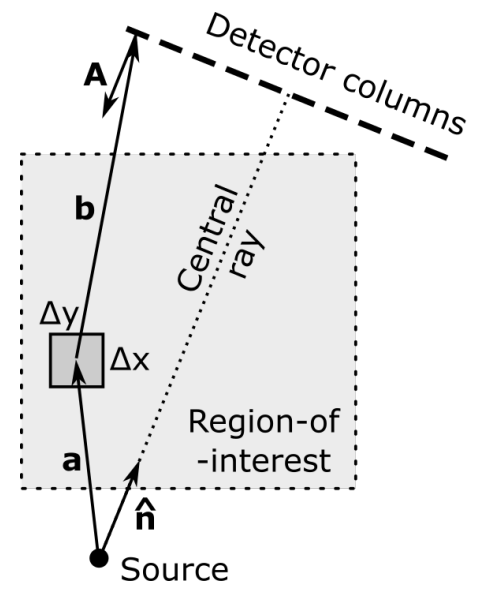

Fig. 1. The top view of the scattering geometry. The symbols in the figure are: $\mathbf{a}=$ source-to-voxel vector, $\boldsymbol{b}=$ voxel-to-detector vector, $\boldsymbol{\Delta} \boldsymbol{x}$ and $\boldsymbol{\Delta} \boldsymbol{y}$ are the voxel dimensions in the horizontal plane. The anode plane that emits X-ray photons is oriented along the unit vector $\hat{\mathbf{n}}$, whereas the detector pixel surface is given by the oriented surface area $\mathbf{A}$ in units of $\mathrm{mm}^{2}$.

\section{A. Source trajectory}

While many source trajectories are possible (such as an irregular polygon inscribed within the available tunnel space), in this article we consider a generic example, which is a circular trajectory. Likewise, the detector could have irregular or curved shape as well, but here we only show an example with a flat panel detector. The region-of-interest (ROI) is a square of size $L_{x}=L_{y}=200 \mathrm{~mm}$. We assume that the phantom has constant composition along the third axis $\mathbf{z}$, which is a useful approximation if the illuminated fan beam is thinner than the objects in the phantom. For computational convenience, the origin of the Cartesian coordinate system is chosen in the left bottom corner of the ROI. The plane in which the source rotates defines the origin of the $z$-axis. The trajectory of the source is thus given by

$$
\mathbf{R}_{\text {src }}=\left(L_{x} / 2+\rho_{\text {src }} \sin \alpha\right) \hat{\mathbf{x}}+\left(L_{y} / 2-\rho_{\text {src }} \cos \alpha\right) \hat{\mathbf{y}}
$$

where $\rho_{\text {src }}=150 \mathrm{~mm}$ is the distance from the source to the middle of the ROI and $\alpha=2 \pi(\mathrm{src} / \mathrm{SRC})$ is the view angle, with $\operatorname{src}=0,1, \ldots,(\mathrm{SRC}-1)$ being the source index. Unit vectors in this article are denoted in bold and with a hat, for example $\hat{\mathbf{x}}=\mathbf{x} /|\mathbf{x}|$.

The detector trajectory is given by

$$
\mathbf{R}_{\mathrm{det}}=\left(L_{x} / 2-\rho_{\mathrm{det}} \sin \alpha+c \cos \alpha\right) \hat{\mathbf{x}}+\left(L_{y} / 2+\rho_{\operatorname{det}} \cos \alpha+c \sin \alpha\right) \hat{\mathbf{y}}+z_{0} \hat{\mathbf{z}}
$$

where $c=(\mathrm{col}-\mathrm{COL} / 2+0.5) *($ column pitch) is the position of the detector column with respect to the central ray. The radius of the detector trajectory is $\rho_{\text {det }}=170 \mathrm{~mm}$. In this work the detector column pitch is chosen to be $0.5 \mathrm{~mm}$, while the height of the first detector row is $z_{0}=10 \mathrm{~mm}$ (see Fig. 2). In reality, due to the finite source size, some part of the transmitted beam can reach above the $z=0$ plane, hence the first row must have enough vertical offset to avoid it. At the same time, the diffraction detectors should be as close to the direct beam as possible, to capture the lowest $q$ photons where many XRD peaks can often be found, due to the high photon energy $E$ (see Eq. (12)).

The vector normal to the detector surface area is given by

$$
\hat{\mathbf{A}}=\hat{\mathbf{x}} \sin \alpha-\hat{\mathbf{y}} \cos \alpha
$$

whereas the source surface area (the anode) is perpendicular to

$$
\hat{\mathbf{n}}=-\hat{\mathbf{x}} \sin \alpha \sin \beta+\hat{\mathbf{y}} \cos \alpha \sin \beta-\hat{\mathbf{z}} \cos \beta
$$

where $\beta=30^{\circ}$ is the tilt of the anode plane.

\section{B. Scattering geometry}

The geometry factor $G$ in Eq. (2) is composed of the following terms:

$$
G=\underbrace{\Delta x \Delta y \Delta z}_{\text {volume }} \underbrace{|\mathbf{a}|^{-2}}_{\text {fluence }} \underbrace{\left(1+\cos ^{2} \theta\right) / 2}_{\text {polarization }} \underbrace{\Delta \Omega}_{\text {solid angle }}
$$

Let us consider one scattering pathway, which is a triangle between the source, the voxel, and the detector, as shown in Figs. 1 and 2 . The source-to-voxel vector is $\mathbf{a}$ and the voxel-to-detector vector is $\mathbf{b}$. The in-plane area of the voxel $\Delta x \Delta y$ is the discretization chosen by us, and has to be sufficiently small to capture the spatial features of the phantom. We use 


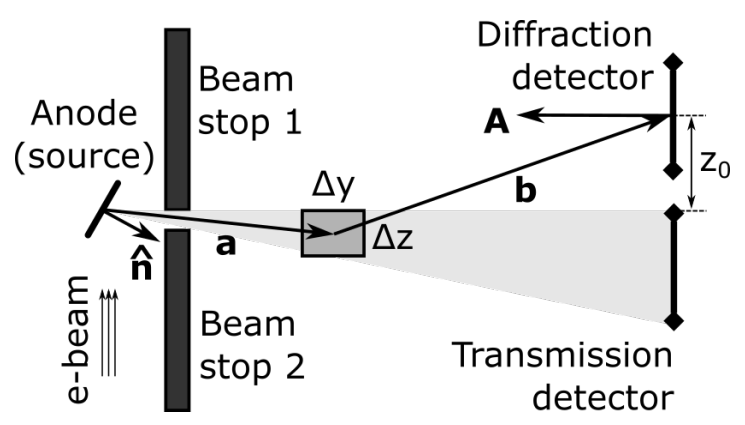

Fig. 2. The side view of the scattering geometry. The symbols in the figure are: $\mathbf{a}=$ source-to-voxel vector, $\boldsymbol{b}=$ voxel-to-detector vector, $\boldsymbol{\Delta} \boldsymbol{z}=$ illuminated voxel thickness in along the tunnel $(\boldsymbol{z})$ axis, $\boldsymbol{\Delta} \boldsymbol{y}=$ an in-plane width of the voxel. The anode plane that emits X-ray photons is oriented along the unit vector $\hat{\mathbf{n}}$, whereas the detector pixel surface is given by the oriented surface area $\mathbf{A}$ in units of $\mathrm{mm}^{2}$. The first row of the diffraction detector panel is shown, with its center position at a height $z_{0}$ above the plane of the source.

$\Delta x=\Delta y=1 \mathrm{~mm}$ in our example. The thickness of the illuminated slice (see gray area in Fig. 2) is determined by the beamstop opening. We assume that the phantom is uniform along the $z$-axis, and that the illumination thickness is sufficiently small that only a single layer of voxels gives acceptable numerical accuracy. The top edge of the illuminated wedge is set at $\phi_{1}=0^{\circ}$, while the bottom edge is slanted at an angle $\phi_{2}=-0.5^{\circ}$, but these settings can be tuned to optimize the flux and resolution of the scanner for specific applications. The voxel thickness is thus given by

$$
\Delta z=\left(\frac{a_{x} n_{x}+a_{y} n_{y}}{\sqrt{n_{x}^{2}+n_{y}^{2}}}\right)\left(\tan \phi_{1}-\tan \phi_{2}\right)
$$

The position of the middle of the voxel along the $z$-axis is:

$$
v_{z}=\left(\frac{a_{x} n_{x}+a_{y} n_{y}}{\sqrt{n_{x}^{2}+n_{y}^{2}}}\right)\left(\frac{\tan \phi_{1}+\tan \phi_{2}}{2}\right)
$$

This position is used to compute the $z$ components of both $\mathbf{a}$ and $\mathbf{b}$. The inverse square length $|\mathbf{a}|^{-2}$ accounts for the photon fluence (number of photons per surface area) at the location of the voxel. Notice that the voxel thickness, Eq. (8), increases linearly with the source-to-voxel distance. Together with the $|\mathbf{a}|^{-2}$ fluence factor, the number of diffracted photons goes down as $|\mathbf{a}|^{-1}$.

The angle between the two legs is $\cos \theta=\hat{\mathbf{a}} \cdot \hat{\mathbf{b}}$. It is used to compute the polarization factor $\left(1+\cos ^{2} \theta\right) / 2$, which applies for non-polarized X-ray sources like vacuum tubes [21]. Finally, from the vantage point of the voxel, a detector of oriented surface area $\mathbf{A}$ subtends a solid angle

$$
\Delta \Omega=\frac{\mathbf{A} \cdot \mathbf{b}}{|\mathbf{b}|^{3}}
$$

The overall units of the geometry factor $G$ are $\mathrm{mm}$.

\section{A small, but finite scattering pathway}

Diffraction patterns of high-quality crystals often have very sharp, narrow, peaks (see Fig. 4. To measure them, the experimental uncertainty of the wavevector transfer $q$ should be substantially smaller than the intrinsic peak width. The $q$-resolution of our XRD tomography setup will be quite low compared to that of dedicated lab diffractometers, hence our reconstructed patterns will be smeared out. To quantify the smearing, in this chapter we derive the resolution formula for a realistic, finite-sized scattering pathway, as shown in Fig. 3 . The accuracy of $q$ is determined by the size of the source and the voxel, as well as the size and energy sensitivity of the detector.

The average wavevector transfer for a photon of energy $E$, traveling from the center of the source to the center of the voxel, and on to the center of the detector, is given by

$$
\mathbf{q}=\frac{E}{\hbar c}(\hat{\mathbf{b}}-\hat{\mathbf{a}})
$$

The amplitude is

$$
q=|\mathbf{q}|=\frac{2 E}{\hbar c}(1-\hat{\mathbf{a}} \cdot \hat{\mathbf{b}})=\frac{2 E}{\hbar c} \sin (\theta / 2)
$$

Here $\hbar c=1.973 \mathrm{keV} \AA$ is the Planck's constant multiplied by the speed of light. Since all three locations have a finite size, the wavevector transfer also has a finite distribution around the mean. For example, consider a scatterer located a small vector 


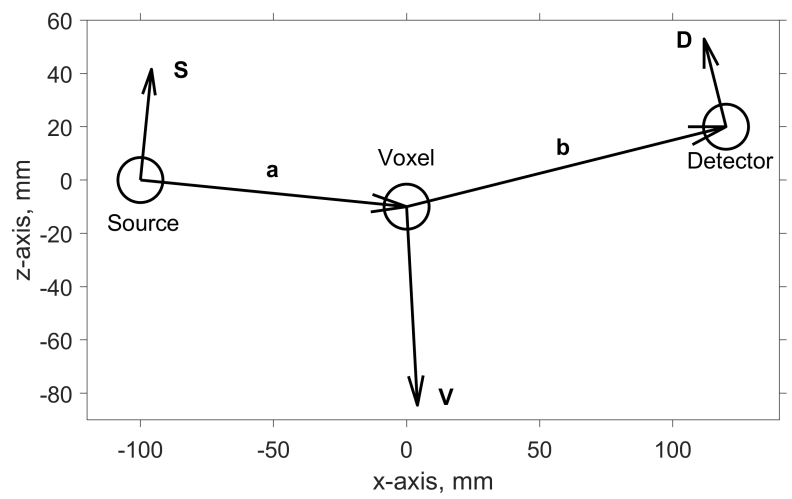

Fig. 3. A typical scattering geometry, defined by the incoming $\hat{a}$ and outgoing $\hat{\mathbf{b}}$ wave vectors. For the sake of generality, the two vectors are unequal. We also show the principal directions $\mathbf{S}, \mathbf{V}$, and $\mathbf{D}$ which cause the main uncertainty $\boldsymbol{\Delta} \boldsymbol{q}$ (see Eq. (27)). The large circles illustrate the finite-sized source, voxel, and detector regions. Note that in general the three regions can have arbitrary, non-circular shapes as described in Eqs. 29- 32 . Specific points within those regions are labeled with vectors s, v, and d, respectively (not shown).

$\mathbf{v}$ away from the center of the voxel. The difference of the incoming wave direction is

$$
\begin{aligned}
\Delta \hat{\mathbf{a}} & =\frac{\mathbf{a}+\mathbf{v}}{\sqrt{(\mathbf{a}+\mathbf{v})^{2}}}-\frac{\mathbf{a}}{\sqrt{\mathbf{a}^{2}}} \\
& =\frac{1}{a}\left[(\mathbf{a}+\mathbf{v})\left(1-\frac{\mathbf{a} \cdot \mathbf{v}}{a^{2}}+\mathcal{O}(v / a)^{2}\right)-\mathbf{a}\right] \\
& =\frac{\mathbf{v}-\hat{\mathbf{a}}(\hat{\mathbf{a}} \cdot \mathbf{v})}{a}+\mathcal{O}(v / a)^{2}
\end{aligned}
$$

Next, allow the source and the detector to have finite sizes too, described by small vectors $\mathbf{s}$ and $\mathbf{d}$ pointing away from their respective centers. In other words, an arbitrary off-center scattering pathway is composed of two modified legs:

$$
\begin{aligned}
& \mathbf{a} \rightarrow \mathbf{a}+(\mathbf{v}-\mathbf{s}) \\
& \mathbf{b} \rightarrow \mathbf{b}+(\mathbf{d}-\mathbf{v})
\end{aligned}
$$

Generalizing Eq. (13) and ignoring second-order terms in $s, v$, and $d$, we find the full change of the wavevector transfer when the scattering pathway is slightly misplaced:

$$
\Delta \mathbf{q}=\frac{e}{E} \mathbf{q}+\frac{E}{\hbar c}\left\{\frac{(\mathbf{d}-\mathbf{v})-[\hat{\mathbf{b}} \cdot(\mathbf{d}-\mathbf{v})] \hat{\mathbf{b}}}{b}-\frac{(\mathbf{v}-\mathbf{s})-[\hat{\mathbf{a}} \cdot(\mathbf{v}-\mathbf{s})] \hat{\mathbf{a}}}{a}\right\}
$$

where $e$ is a small deviation from the average pathway energy $E$. For isotropic materials, only the magnitude of the change is important:

$$
\begin{aligned}
\Delta q & =\sqrt{(\mathbf{q}+\Delta \mathbf{q})^{2}}-q \\
& =q\left[1+\frac{\mathbf{q} \cdot \Delta \mathbf{q}}{q^{2}}+\mathcal{O}(\Delta q / q)^{2}\right]-q \\
& =\frac{\mathbf{q} \cdot \Delta \mathbf{q}}{q}+\mathcal{O}(\Delta q / q)^{2}
\end{aligned}
$$

Neglecting terms of order $(\Delta q)^{2}$ and higher, the dot product becomes

$$
\begin{aligned}
\Delta q & =\frac{e}{E} q+\left(\frac{E}{\hbar c}\right)^{2}\left(\frac{\mathbf{s} \cdot \mathbf{S}+\mathbf{v} \cdot \mathbf{V}+\mathbf{d} \cdot \mathbf{D}}{q a b}\right) \\
& =\frac{2 e}{\hbar c} \sin (\theta / 2)+\frac{E}{\hbar c}\left(\frac{\mathbf{s} \cdot \mathbf{S}+\mathbf{v} \cdot \mathbf{V}+\mathbf{d} \cdot \mathbf{D}}{2 a b \sin (\theta / 2)}\right)
\end{aligned}
$$


where we have defined three auxiliary vectors

$$
\begin{aligned}
\mathbf{S} & =\mathbf{b}-\hat{\mathbf{a}}(\hat{\mathbf{a}} \cdot \mathbf{b}) \\
\mathbf{D} & =-\mathbf{a}+\hat{\mathbf{b}}(\hat{\mathbf{b}} \cdot \mathbf{a}) \\
\mathbf{V} & =-(\mathbf{S}+\mathbf{V})
\end{aligned}
$$

The key quantity for computing realistic diffraction signals is the mean square width of a given scattering pathway:

$$
\left\langle(\Delta q)^{2}\right\rangle=\left(\frac{2 \sin (\theta / 2)}{\hbar c}\right)^{2}\left\langle e^{2}\right\rangle+\left(\frac{E}{\hbar c}\right)^{2}\left(\frac{\left\langle(\mathbf{s} \cdot \mathbf{S})^{2}\right\rangle+\left\langle(\mathbf{v} \cdot \mathbf{V})^{2}\right\rangle+\left\langle(\mathbf{d} \cdot \mathbf{D})^{2}\right\rangle}{[2 a b \sin (\theta / 2)]^{2}}\right)
$$

It contains four contributions, which is the range of energies, as well as non-zero sizes of the source, the voxel, and the detector. For sufficiently small bins, we can assume that the energy distribution within each energy bin is constant (uniform), resulting in

$$
\left\langle e^{2}\right\rangle=\frac{1}{\Delta E} \int_{-\Delta E / 2}^{\Delta E / 2} e^{2} d e=\frac{(\Delta E)^{2}}{12}
$$

The size of the source refers to the spatial distribution of points that emit X-rays. In general, it is a 3D scalar function $\rho(\mathbf{s})$ (assumed to be normalized $\int \rho(\mathbf{s}) d \mathbf{s}=1$ ) and the relevant moment for us is

$$
\left\langle(\mathbf{s} \cdot \mathbf{S})^{2}\right\rangle=\int \rho(\mathbf{s})(\mathbf{s} \cdot \mathbf{S})^{2} d \mathbf{s}
$$

In this paper we do not investigate the detailed shape of $\rho(\mathbf{s})$, and instead assume a simplified model, namely a flat square patch of the anode surface area whose normal vector is given by $\hat{\mathbf{n}}$. In this case, Eq. (28) generalizes to

$$
\left\langle(\mathbf{s} \cdot \mathbf{S})^{2}\right\rangle=\frac{(\Delta s)^{2}}{12}\left(\mathbf{S}^{2}-(\mathbf{S} \cdot \hat{\mathbf{n}})^{2}\right)=\frac{(\Delta s)^{2}}{12}|\mathbf{S} \times \hat{\mathbf{n}}|^{2}
$$

where $\Delta s=0.5 \mathrm{~mm}$ is the side length of the rectangular patch that emits X-rays, a.k.a the focal spot. The same formula also applies for the detector, which we set to be a rectangle of surface area $|\mathbf{A}|=0.5 \mathrm{~mm}^{2}$ :

$$
\left\langle(\mathbf{d} \cdot \mathbf{D})^{2}\right\rangle=\frac{|\mathbf{D} \times \mathbf{A}|^{2}}{12 A}
$$

Lastly, the voxel is modeled as a rectangular parallelepiped of uniform density, in which case the formula generalizes to

$$
\left\langle(\mathbf{v} \cdot \mathbf{V})^{2}\right\rangle=\frac{\left(\Delta x V_{x}\right)^{2}+\left(\Delta y V_{y}\right)^{2}+\left(\Delta z V_{z}\right)^{2}}{12}
$$

\section{Diffraction patterns}

The vast majority of XRD studies report the scattered intensity in arbitrary units. This is adequate for determining crystal structures, which can be inferred solely from the positions and relative intensities of the diffraction peaks. On the other hand, a key consideration for an XRD imaging system is the photon flux, which in turn depends on the scattering cross-sections in absolute units. This step is crucial to design a system that strikes a fair balance between the flux and the accuracy of the wavevector transfer $q$, which is needed to resolve individual XRD peaks. Currently, intensity calibration is experimentally feasible only for a limited number of situations. In particular, small angle X-ray scattering (SAXS) machines are sometimes calibrated to an absolute scale, since in that geometry the Ewald sphere can be assumed as flat and the detector plane can be perfectly matched with it. In wide angle X-ray scattering (WAXS), this assumption is not applicable and an additional correction is required for the angle it makes with the tangent of the sphere surface. Due to this and other complications, most authors do not attempt to calibrate their WAXS data, let alone XRD. At present, there exist several studies performed using the same sample on both SAXS and WAXS machines having an overlap in their $q$-ranges, which allows a straightforward cross-calibration of the two datasets. Available examples include silver behenate [23], NiBpene flexible MOFs (metal-organic frameworks) [24], and isotactic polypropylene [25], [26].

For general materials of interest to security, healthcare, and manufacturing, absolute XRD intensity calibration is not readily available. On the other hand, if we know the atomic composition, we can compute the theoretical Rayleigh ( $R$, coherent) and Compton $(C$, incoherent) scattering on an absolute scale:

$$
\begin{aligned}
& \left(\frac{1}{V} \frac{d \sigma}{d \Omega}\right)_{\text {Rayleigh }}=r_{e}^{2} N_{A} \rho\left(\frac{N_{1} R_{1}^{2}+N_{2} R_{2}^{2}+\cdots}{N_{1} M_{1}+N_{2} M_{2}+\cdots}\right) \\
& \left(\frac{1}{V} \frac{d \sigma}{d \Omega}\right)_{\text {Compton }}=r_{e}^{2} N_{A} \rho\left(\frac{N_{1} C_{1}+N_{2} C_{2}+\cdots}{N_{1} M_{1}+N_{2} M_{2}+\cdots}\right)
\end{aligned}
$$




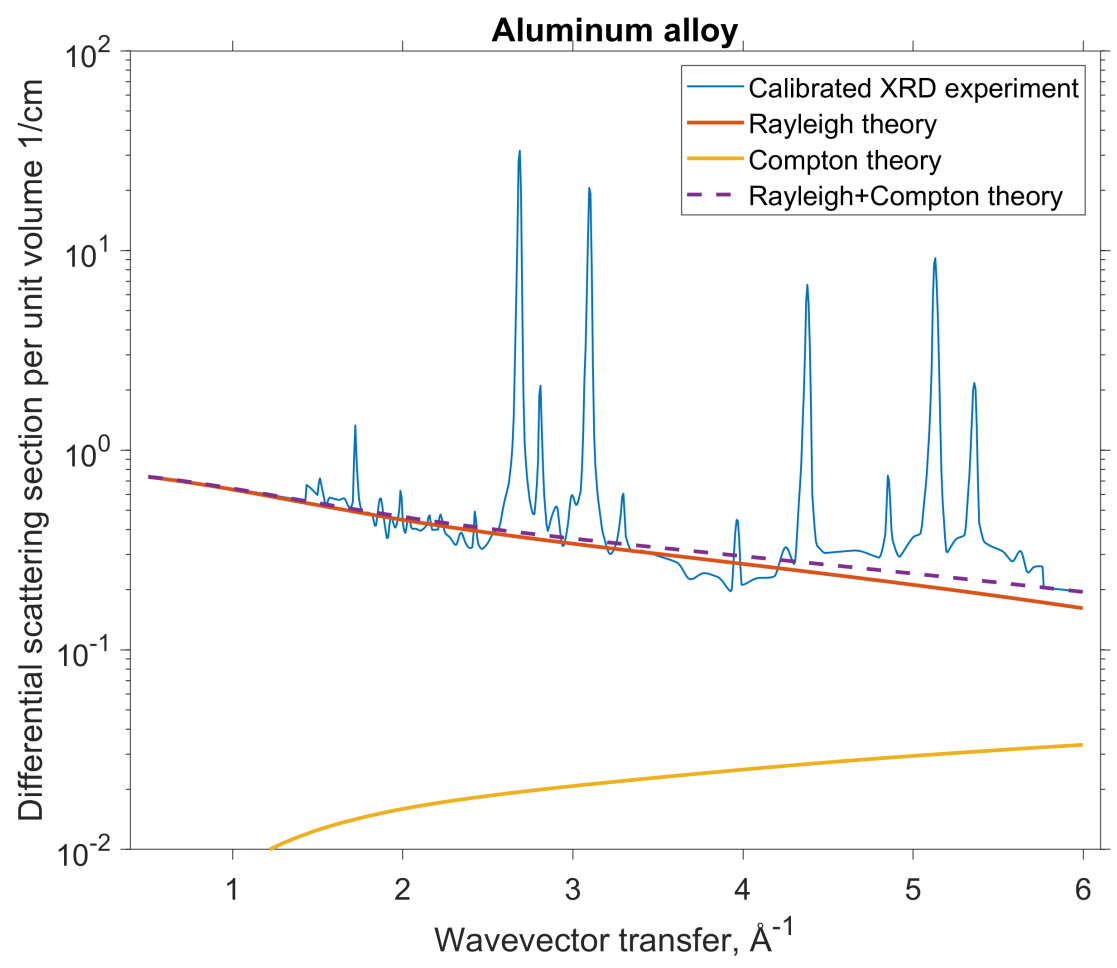

Fig. 4. Experimental XRD pattern of an aluminum alloy [22], rescaled by us to an absolute scale of $\mathrm{cm}^{-1}$ to roughly match the theoretical, nondiffracting scattering, composed of Compton and Rayleigh terms. If experimental XRD data does not fully cover our desired range of $0.5-6 \AA^{-1}$, we use the theoretical data for a non-diffracting material of the same composition.

Here $\rho$ is the mass density, $N_{1,2, \ldots}$ is the number of a given atomic species and $M_{1,2, \ldots}$ is the atomic weight. The classical electron radius is $r_{e}=2.818 \times 10^{-15} \mathrm{~m}$, while the Avogadro's number is $N_{A}=6.022 \times 10^{23} \mathrm{~mol}^{-1}$. The $R$ and $C$ scattering cross-sections for all atoms are tabulated in Ref. [27]. The above expressions would apply if the atoms were distributed randomly in space. In real materials, the atomic structure is far from random, which results in constructive interference peaks and destructive interference valleys in the Rayleigh component. Roughly, the bottom of the measured XRD signal can reach as low as theoretical Compton scattering, while the baseline of the peaks (the inflection point) may roughly be where the theoretical Rayleigh+Compton curve is. Using these rules-of-thumb we have the adjusted the amplitude of aluminum XRD data as shown in Fig. 4 . We could also assess the validity of our rule-of-thumb in one case where the experimentally calibrated data was available (isotactic polypropylene [26], not shown), and the two curves were well within the same order-of-magnitude.

As a side note, we mention that the polarization factor $\left(1+\cos ^{2} \theta\right) / 2$ is often not accounted for in published XRD data. Since the data is often collected using low energy X-rays, we have to divide it by its own polarization factor, before using the data in our simulations. This correction can be significant, up to a factor of 2, and is important to include because our XRD imaging scanner uses X-rays of much higher energy, hence the diffraction peaks at a given $q$ value appear at substantially smaller scattering angles $\theta$.

For computing the ground truth (forward projection), the cross-section in Eq. 2) is obtained by smearing the high-resolution XRD data with the width of the scattering pathway from Eq. 27):

$$
F(q)=\int_{0}^{\infty}\left(\frac{1}{V} \frac{d \sigma}{d \Omega}\left(q^{\prime}\right)\right)_{\mathrm{XRD}} \frac{d q^{\prime}}{\sqrt{2 \pi\left\langle(\Delta q)^{2}\right\rangle}} \exp \left(-\frac{\left(q^{\prime}-q\right)^{2}}{2\left\langle(\Delta q)^{2}\right\rangle}\right)
$$

\section{E. Source spectrum}

Transmission tomography usually operates with hard X-rays in the $100-200 \mathrm{keV}$ range, which have a weak attenuation needed to penetrate thick materials. X-ray diffraction, on the other hand, is typically done on very small samples in a laboratory setting, and using much softer X-rays, for example $8.04 \mathrm{keV}$ which is the copper $\mathrm{K}-\alpha$ line. For small samples the beam attenuation is not important, and a lower X-ray energy is preferred as it broadens the spacing of diffraction peaks seen on the detector (see Equation (12), hence increasing the resolution. Our combined transmission+diffraction scanner should strike some middle ground between these two extremes. The optimal spectrum will depend on the specific application, so in this paper we just pick a reasonable value of $80 \mathrm{keV}$ for the anode voltage and a default $1 \mathrm{~mm}$ aluminum filter to remove the lowest energy photons, see Fig. 5. The spectrum values $\Phi(E)$ provided by SpekCalc refer to the number of photons per $\mathrm{cm}^{2}$ at a reference distance 


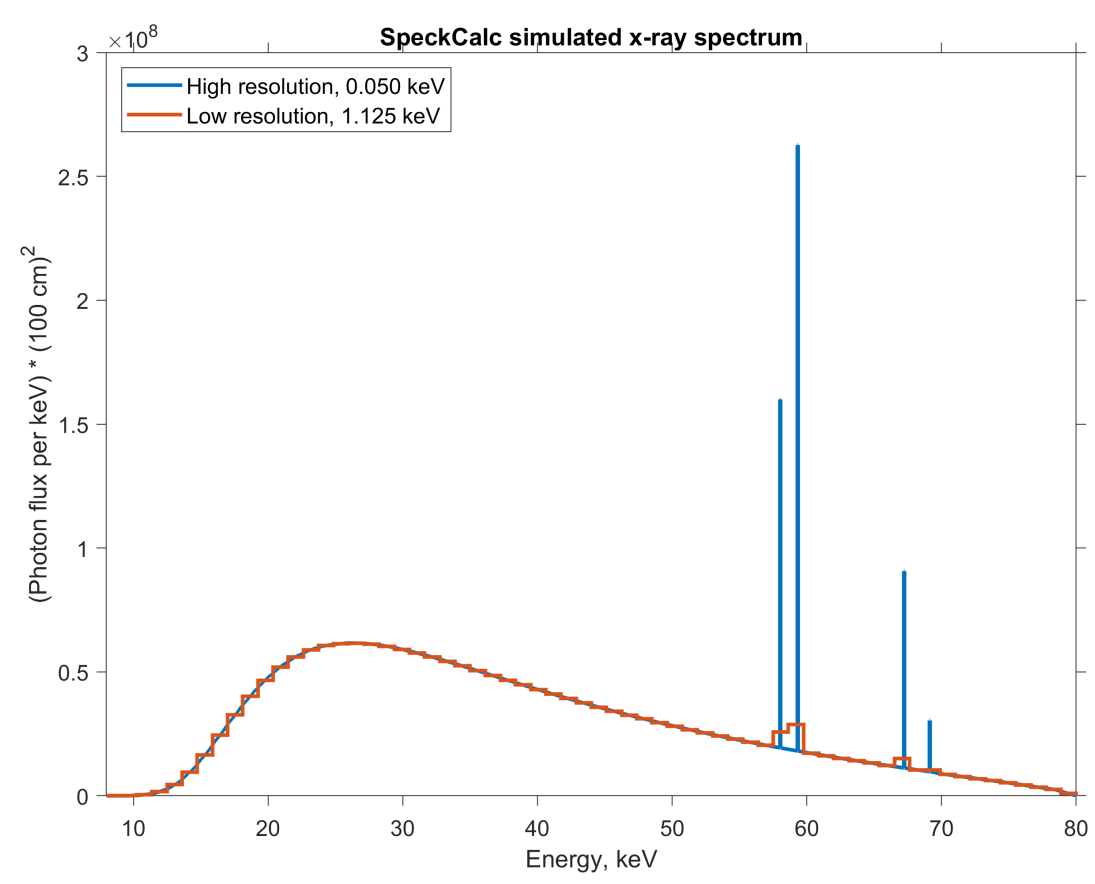

Fig. 5. Source spectrum simulation from SpekCalc. The number of photons is proportional to the source current and exposure time, which we have assumed to be $10 \mathrm{~mA}$ and $0.1 \mathrm{~ms}$, or $1 \mathrm{mC}$ of electron charge.

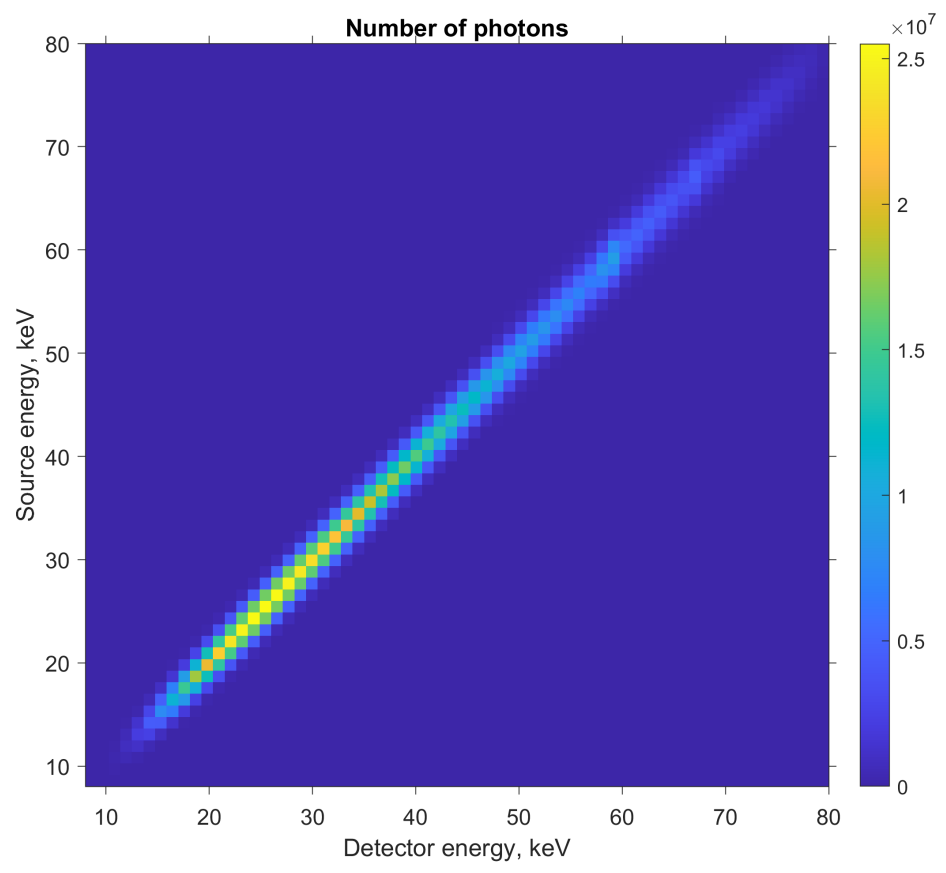

Fig. 6. Convolution of detector response with the source spectrum. Due to rapid drop-off away from the diagonal, we use only 5 bins to the left and right to sum over the spectrum (see Eq. (2)), hence 11 terms in total, rather than all 64.

$a_{0}=100 \mathrm{~cm}$, per exposure time, per source current. The absolute number of photons is proportional to the time of exposure which we set to $0.1 \mathrm{~ms}$ and to the source current which we set to $10 \mathrm{~mA}$, but these settings can vary wildly between different applications of XRD imaging. We have also multiplied the flux by $a_{0}^{2}$, so it becomes a dimensionless number of photons, per energy bin width, shown in Fig. 5. Anisotropic features of the spectrum such as the heel effect are not considered in this work, but would be straightforward to implement within the current algorithm.

This high-resolution spectrum will never be observed with a realistic energy-sensitive detector, which has a resolution 


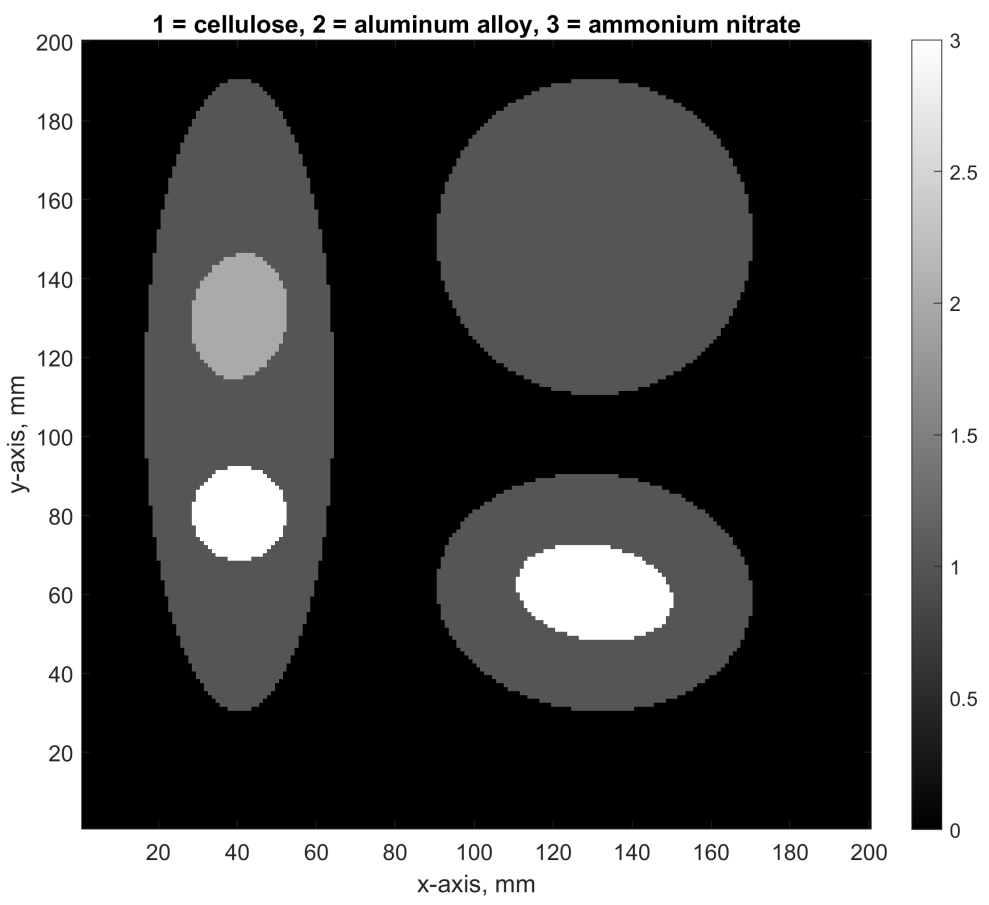

Fig. 7. The multi-material phantom used in this work. Each of the three materials is colored a different shade of gray.

of roughly $\sigma=\left(1.61 \mathrm{keV}+0.025 E_{\mathrm{src}}\right) / 2$, as detailed in experimental [28] and theoretical [29] studies. Quite a few years have passed since the publication of these papers, hence we have taken the liberty to assume that the energy resolution has been improved by a factor of two, or will be so in the near future. We discretize the spectrum to a reasonable number of NRG $=64$ energy bins, spaced equidistantly between $E_{\min }=8 \mathrm{keV}$ and $E_{\max }=80 \mathrm{keV}$, with a bin width $\Delta E=$ $\left(E_{\max }-E_{\min }\right) / \mathrm{NRG}=1.125 \mathrm{keV}$, which is on the lower end of the energy resolution range $\sigma=0.905-1.805 \mathrm{keV}$. The source energy bins are labeled with the index $\operatorname{nrgsrc}=1,2, \ldots, \mathrm{NRG}$, and similarly the detector energy bins are labeled with the index nrgdet $=1,2, \ldots$, NRG. We can then compute the number of photons expected at a given detector bin, due to illumination from each of the source bins:

$$
\eta(\mathrm{nrgsrc}, \mathrm{nrgdet})=\int_{E_{1}}^{E_{2}} d E_{\mathrm{src}} \int_{E_{3}}^{E_{4}} d E_{\mathrm{det}} \frac{\Phi\left(E_{\mathrm{src}}\right) a_{0}^{2}}{\sqrt{2 \pi \sigma^{2}}} \exp \left(-\frac{\left(E_{\mathrm{src}}-E_{\mathrm{det}}\right)^{2}}{2 \sigma^{2}}\right)
$$

The limits of integration (the bin edges) are given by

$$
\begin{aligned}
& E_{1}=E_{\min }+(\operatorname{nrgsrc}-1) \Delta E \\
& E_{2}=E_{\min }+\operatorname{nrgsrc} \Delta E \\
& E_{3}=E_{\min }+(\operatorname{nrgdet}-1) \Delta E \\
& E_{4}=E_{\min }+\operatorname{nrgdet} \Delta E
\end{aligned}
$$

The result of Eq. 36 is a dimensionless number of photons and is shown in Fig. 6 In other words, $\eta$ (nrgsrc, nrgdet) is the convolution of the source spectrum and the detector efficiency, for all bin pairs.

\section{F. Beam attenuation}

The phantom used in this work is shown in Fig. 7 and corresponds to the gray area in Fig. 1 It consists of $(\mathrm{NX}=$ $200) \times(\mathrm{NY}=200)$ voxels of size $1 \mathrm{~mm}^{2}$. We assume that multi-energy computed tomography (MECT) has been performed for this phantom, using the transmission detector data, see the transmission detectors in Fig. 2 The result of MECT is X-ray attenuation coefficient as a function of energy at each point

$$
\mathbf{r}=[(\mathrm{nx}-0.5) \Delta x,(\mathrm{ny}-0.5) \Delta y]
$$

of the phantom, which can be expressed, for example, as a sum of photoelectric $\left(a_{1}\right)$ and Compton $\left(a_{2}\right)$ coefficients:

$$
\mu(\mathbf{r}, E)=a_{1}(\mathbf{r}) f_{1}(E)+a_{2}(\mathbf{r}) f_{2}(E)
$$


The energy basis functions are given by

$$
\begin{aligned}
& f_{1}=\varepsilon^{-m} \\
& f_{2}=\frac{1+\varepsilon}{\varepsilon^{2}}\left[\frac{2(1+\varepsilon)}{1+2 \varepsilon}-\frac{\log (1+2 \varepsilon)}{\varepsilon}\right]+\frac{\log (1+2 \varepsilon)}{2 \varepsilon}-\frac{1+3 \varepsilon}{(1+2 \varepsilon)^{2}}
\end{aligned}
$$

where $m=3$ is the photoelectric exponent and $\varepsilon=E / m c^{2}$ is the dimensionless photon energy (i.e. divided by the electron rest energy $m c^{2}$ ). In this paper we make an optimistic assumption that MECT reconstruction is ideal, in which case the coefficients for each material should be the same as

$$
\begin{aligned}
& a_{1}=K_{1} \rho \frac{N_{1} Z_{1}^{n}+N_{2} Z_{2}^{n}+\cdots}{N_{1} M_{1}+N_{2} M_{2}+\cdots} \\
& a_{2}=K_{2} \rho \frac{N_{1} Z_{1}+N_{2} Z_{2}+\cdots}{N_{1} M_{1}+N_{2} M_{2}+\cdots}
\end{aligned}
$$

Here $n=4.2$ and $K_{1}=1.047 \times 10^{-7} \mathrm{~cm}^{2} / \mathrm{mol}$ are empirical parameters, while $K_{2}=2 \pi r_{e}^{2} N_{A}=0.30 \mathrm{~cm}^{2} / \mathrm{mol}$ is the Compton parameter that can be computed from the classical electron radius $r_{e}$ and the Avogadro's number $N_{A}$. Other symbols are:

- $\rho$ is the mass density of the material in $\mathrm{g} / \mathrm{cm}^{3}$,

- $N_{1,2, \ldots}$ is the number of atoms,

- $Z_{1,2, \ldots}$ is the atomic number, and

- $M_{1,2, \ldots}$ is the atomic weight.

For example, the chemical composition of ammonium nitrate is $\mathrm{NH}_{4} \mathrm{NO}_{3}$, which in our notation is $Z=[1,7,8], N=[4,2,3]$, and $M=[1.00784,14.0067,15.999] \mathrm{g} \mathrm{mol}^{-1}$.

The photon survival probabilities mentioned in Eq. (2) are given by:

$$
\begin{gathered}
P_{\text {in }}(E)=\exp \left(-f_{1}(E) \int_{\text {src }}^{\text {vox }} a_{1}(\mathbf{r}) d r-f_{2}(E) \int_{\text {src }}^{\text {vox }} a_{2}(\mathbf{r}) d r\right) \\
P_{\text {out }}(E)=\exp \left(-f_{1}(E) \int_{\text {vox }}^{\operatorname{det}} a_{1}(\mathbf{r}) d r-f_{2}(E) \int_{\text {vox }}^{\operatorname{det}} a_{2}(\mathbf{r}) d r\right)
\end{gathered}
$$

In the simulation, the line integrals are replaced by discrete sums with step size $\Delta r=0.25$ of the voxel size (which is our internal length unit defined as one, i.e. $\Delta x=\Delta y=1$, and all the other lengths are relative to this). In general, the query points along the lines do not match with the locations of the voxels which are on a Cartesian grid, hence we fetch the values for $\left(\mathbf{a}_{1}(\mathbf{r}), \mathbf{a}_{2}(\mathbf{r})\right)$ using bilinear interpolation, and assume them to be zero outside of the region-of-interest (ROI).

\section{G. The forward projector and model matrix}

We now have all the ingredients to carry out the calculation for the expected number of photons, Eq. 22. To perform the inverse calculation, we first have to discretize the $q$-axis to NWT finite-sized bins. Generally, the bins should be somewhat smaller than the intrinsic, physical scanner resolution $\sqrt{\left\langle\Delta q^{2}\right\rangle}$. It is futile to attempt reconstruction at a much finer resolution than that, especially because the matrix size and computational time are already strained and require excessive effort to compute in practice. In this project, we work with NWT $=256$ reconstruction bins, spaced unequally to roughly follow the natural behavior of $\sqrt{\left\langle\Delta q^{2}\right\rangle}$. Furthermore, to speed up calculations, we set the diffraction signal to zero outside of the range $q_{\min }=0.5 \AA^{-1}$ and $q_{\max }=6.0 \AA^{-1}$ in both forward and inverse problems. In reality, a small amount of diffracted photons may reach the detector beyond this range, and we could address it as a general background bias as shown in Eq. (53), but this is beyond the scope of the current work. We define the left edge of our reconstruction bin nwt to be

$$
q_{\text {left }}(\mathrm{nwt})=q_{\min }+\mathrm{nwt} * d q_{0}+\left(q_{\max }-q_{\min }-d q_{0} * \mathrm{NWT}\right) \frac{\mathrm{nwt}^{2}}{\mathrm{NWT}^{2}}
$$

where $d q_{0}=0.01 \AA^{-1}$ is the width of the first bin. The right edge $q_{\text {right }}($ nwt $)$ is the same but with nwt +1 instead of nwt. The average wavevector of the bin is

$$
q_{\text {bin }}(\mathrm{nwt})=\frac{q_{\text {left }}(\mathrm{nwt})+q_{\mathrm{right}}(\mathrm{nwt})}{2}
$$

The $(m, k)$-th element of the matrix $\mathbb{A}$ is the sum of intersections of the reconstruction bin and each physical pathway, namely

$$
A(m, k)=\sum_{\text {vox }=1}^{\text {vox }} G \sum_{\text {nrgsrc }=1}^{\text {NRG }}\left(\frac{q_{\text {right }}-q_{\text {left }}}{\sqrt{2 \pi\left\langle(\Delta q)^{2}\right\rangle}}\right) \exp \left(-\frac{\left(q_{\text {bin }}-q\right)^{2}}{2\left\langle(\Delta q)^{2}\right\rangle}\right) \eta P_{\text {in }} P_{\text {out }}
$$




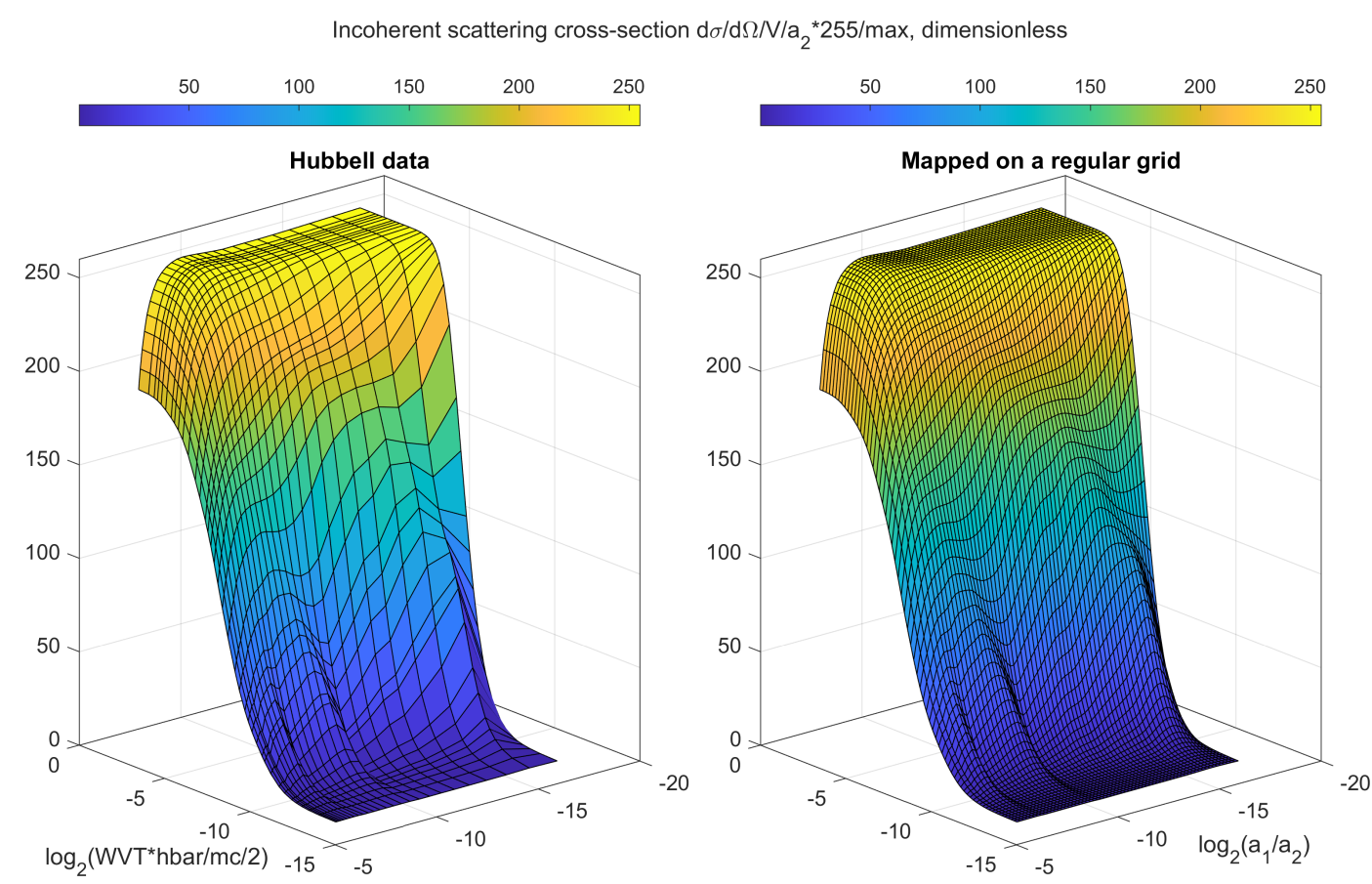

Fig. 8. The map from MECT output $\left(\boldsymbol{a}_{1}, \boldsymbol{a}_{2}\right)$ to the Compton scattering cross-section. The wavevector transfer is expressed in dimensionless units $\boldsymbol{q} \hbar / \boldsymbol{m c} / \mathbf{2}$. Both inputs are on logarithmic axis of base 2 for quicker lookup using a regular $64 \times 64$ grid (right), obtained by interpolating Hubbell's irregularly spaced data (left), available in Ref. [27]. The lookup table is normalized to the range [0,255], so it can be stored as 8 bit integers, to reduce critical shared memory usage.

Notice how the above equation contains every aspect of the scan, except the diffraction pattern $F$. To verify the validity of the matrix, we can multiply it with a column vector (see Eq. (1)) made from bin-averaged scattering cross-sections:

$$
F(k)=\int_{q_{\text {left }}}^{q_{\text {right }}} \frac{d q^{\prime}}{q_{\text {right }}-q_{\text {left }}}\left(\frac{1}{V} \frac{d \sigma}{d \Omega}\left(q^{\prime}\right)\right)_{\mathrm{XRD}}
$$

The resulting photon counts $N(m)$ are close to what we get from direct ground truth summation (Eq. (2)), with small differences due to the finite size of discretization (not shown).

\section{H. Compton scattering background}

A more realistic version of Eq. (1) should include non-zero background (bias) and photon counting noise:

$$
\mathbf{N}=\operatorname{noise}(\mathbb{A} \cdot \mathbf{F}+\text { bias })
$$

There can be many sources of bias, due to imperfect instrumentation (detector blur, lag), as well as secondary X-ray phenomena like scattering. Here we consider bias due to incoherent (Compton) scattering, which may become significant at higher energies and scattering angles. Only a small addition to our previously described XRD calculation is needed to obtain the single-scattered Compton signal as well. Multiple scattering (both coherent, incoherent, and combinations thereof) is beyond the scope of this work, although some progress has been reported in literature in the context of XRD tomography [30]. The number of Compton-scattered photons is computed similarly as in Eq. (2), except that the scattering cross-section $F$ is replaced by the one interpolated from Hubbell's tables, see Fig. 8. It depends on the photoelectric and Compton coefficients $a_{1}$ and $a_{2}$ of each material, which we have already assumed will be available from transmission MECT. We also see that the incoherent scattering cross-section varies smoothly with $q$, hence we do not need to pre-average it (i.e. we can assume that it is constant over the width of the scattering pathway $\sqrt{\left\langle(\Delta q)^{2}\right\rangle}$ ). However, keep in mind that Compton scattering is inelastic, meaning that the electron carries away some of the momentum, which changes the wavevector transfer of the photon:

$$
\frac{\hbar q_{\text {Compton }}}{2 m c}=\left(\frac{\hbar q}{2 m c}\right)\left(\frac{1}{\epsilon}\right) \sqrt{\epsilon+\left(\frac{\hbar q}{2 m c}\right)^{2}}
$$

The above equation is in dimensionless units, and is used to fetch tabulated cross-section data as shown in Fig. 8 . The symbol $\epsilon$ is short for

$$
\epsilon=1+(1-\cos \theta) E_{\text {in }} / m c^{2}
$$


which is also used to calculate the energy of the outgoing photon:

$$
E_{\text {out }}=\frac{E_{\text {in }}}{\epsilon}
$$

We apply the above equation to the left and the right edge of the incoming source photon energy bin, resulting in the two edges of the outgoing energy band, $E_{\text {out } 1}$ and $E_{\text {out2 }}$. We then overlap this range with the detector energy channel range:

$$
\text { overlap }=\frac{\max \left[0, \min \left(E_{\text {out } 2}, E_{\text {det } 2}\right)-\max \left(E_{\text {out } 1}, E_{\text {det } 1}\right)\right]}{E_{\text {out } 2}-E_{\text {out } 1}}
$$

The dimensionless overlap weight above is multiplied with all the terms that enter the sum in Eq. 22. In other words, we perform accumulation with linear interpolation. A simpler, nearest-neighbor approach is also possible, but since our XRD summation code already has all the detector energy channels, we reuse the program structure for Compton as well. Lastly, for Compton scattering, the polarization factor in Eq. (7) has to be replaced by the Klein-Nishina function:

$$
\text { Klein-Nishina }=\epsilon^{2}\left(\frac{\epsilon+\epsilon^{-1}-1+\cos ^{2} \theta}{2}\right)
$$

where $\epsilon=E_{\text {in }} / E_{\text {out }} \geq 1$ is the ratio of the incoming to outgoing energies. In the limit of small energy and/or scattering angle, the Klein-Nishina factor reduces back to the polarization factor $\left(1+\cos ^{2} \theta\right) / 2$.

\section{Results}

Our example phantom (Fig. 7) contains three distinct materials, spread out over six different objects. All objects are fairly large and round, hence we should be able to see their spatial features easily. As a consequence, the transmission MECT data should be very good in this case, allowing for an accurate image segmentation. We have three large objects made from low density cellulose (akin to clothes), and wrapped inside we have a few diffracting objects, one benign (aluminum alloy) and two threats (both ammonium nitrate). The experimental XRD data was digitized from published literature:

- Cellulose is taken from Ref. [31], and we assume density of $0.1 \mathrm{~g} / \mathrm{cm}^{3}$ to be a reasonable model for clothes.

- Aluminum alloy is taken from Ref. [22], using the composition listed in the same paper.

- Ammonium nitrate is taken from Ref. [32]. It is a hazardous material, commonly used as fertilizer, but can be easily detonated, whether intentionally or not.

The results of a noisy experiment with Compton background are shown for one source in Fig. 9 in $2 \mathrm{D}$, and Fig. 10 in $1 \mathrm{D}$. The model matrix $\mathbb{A}$ from Eq. 51] is shown (one slice) in Fig. 11. To reconstruct the unknown diffraction patterns, we apply the iterative Lucy-Richardson algorithm:

$$
\mathbf{F}^{(i+1)}=\left(\frac{\mathbf{F}^{(i)}}{\sum_{m=1}^{M} \mathbb{A}(m, k)}\right) * \mathbb{A}^{T} \cdot\left(\frac{\mathbf{N}}{\mathbb{A} \cdot \mathbf{F}^{(i)}+\text { bias }}\right)
$$

In the above equation, the star $(*)$ symbol and the divisions are point-wise operations, while the dot $(\cdot)$ symbol is the matrixvector product. The initial guess of the iteration, $\mathbf{F}^{(0)}$ could be obtained from Rayleigh scattering, similarly to how we obtained the Compton background (see Fig. 8), but in this work we have just used a constant starting guess. The algorithm guarantees that the solution stays non-negative, and each iteration is guaranteed to reduce the Poisson likelihood cost function, which is appropriate for very low photon counts. Typically, the solution converges within a few hundred iterations. Since we know the Compton background (from transmission MECT results), we can insert it into the denominator of our solver, Eq. (59), which makes it physically more accurate, and also avoids the unstable situation where the denominator is close to zero.

Typical results of our reconstruction are shown for the three materials in Fig. 12 on a logarithmic scale, and Fig. 13 on a linear scale. As anticipated, each reconstructed curve is essentially a low-resolution version of the ground truth. We verify the validity of the solution by multiplying it back with the model matrix, which is shown with red circles in Fig. 10 . The photon counts are close to ground truth calculation.

\section{Discussion}

In this work we have shown how to reconstruct multiple XRD patterns from a large phantom. The setup is essentially the same as fan-beam CT, but with an additional detector on one (or both) sides of the fan. Only diffracted photons can reach those areas, and without a detector, the information contained in them would go to waste. In this design there are no collimators beyond those required by fan-beam CT, resulting in the highest possible XRD photon flux. With transmission alone, MECT for most materials can only yield two numbers per voxel (the photoelectric and Compton coefficients). Our reconstruction, while far from the quality of small sample laboratory XRD, gives much more information than just two numbers, see Fig. 12

Airport security is a particularly demanding area for XRD imaging, because passenger bags often contain multiple diffracting objects. Because of a long list of possible materials, the resolution of the reconstructed patterns is limited and our currently 


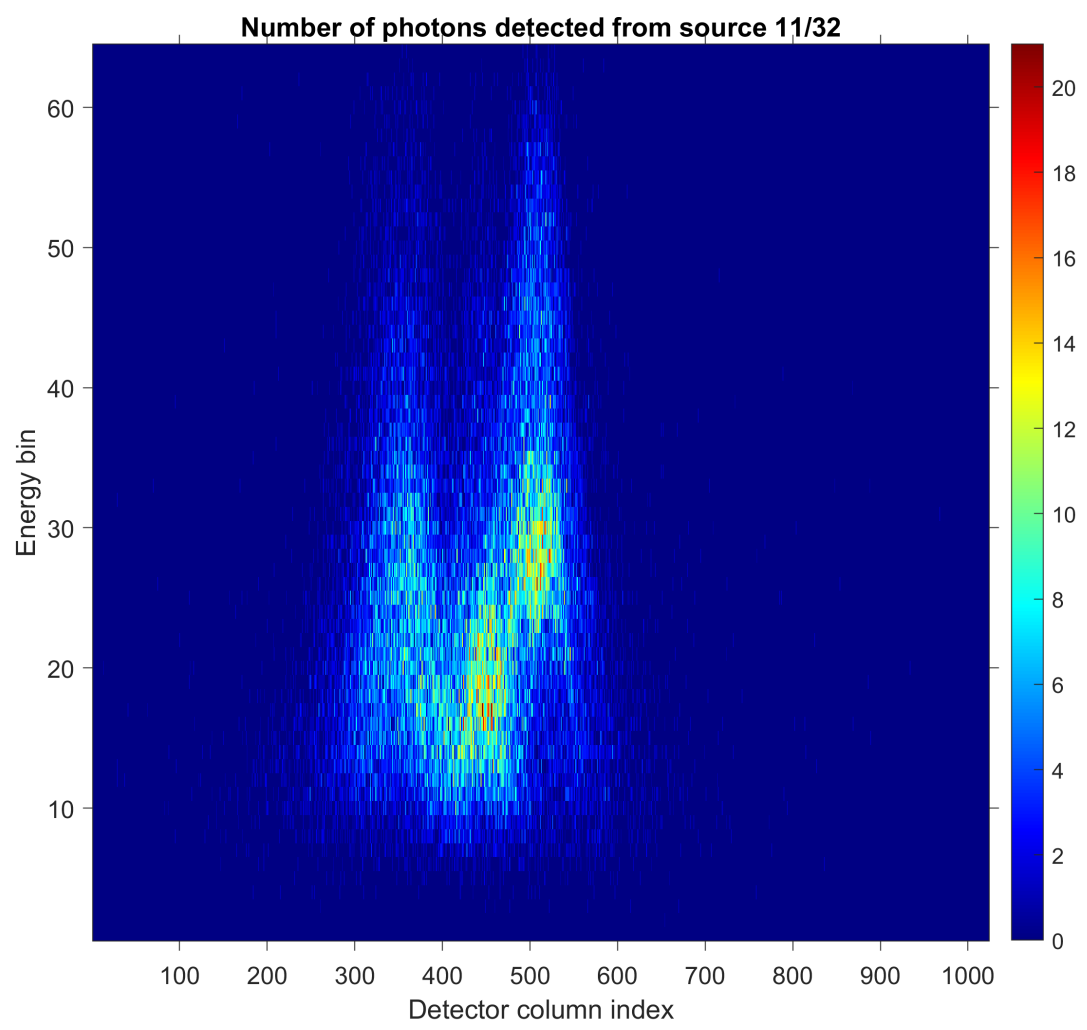

Fig. 9. One slice of the noisy measurement, Eq. 53. Poisson statistics were used to generate the noise. In total there are approximately 44000 photons in this detector snapshot, or about 1100000 photons for all 32 source positions.

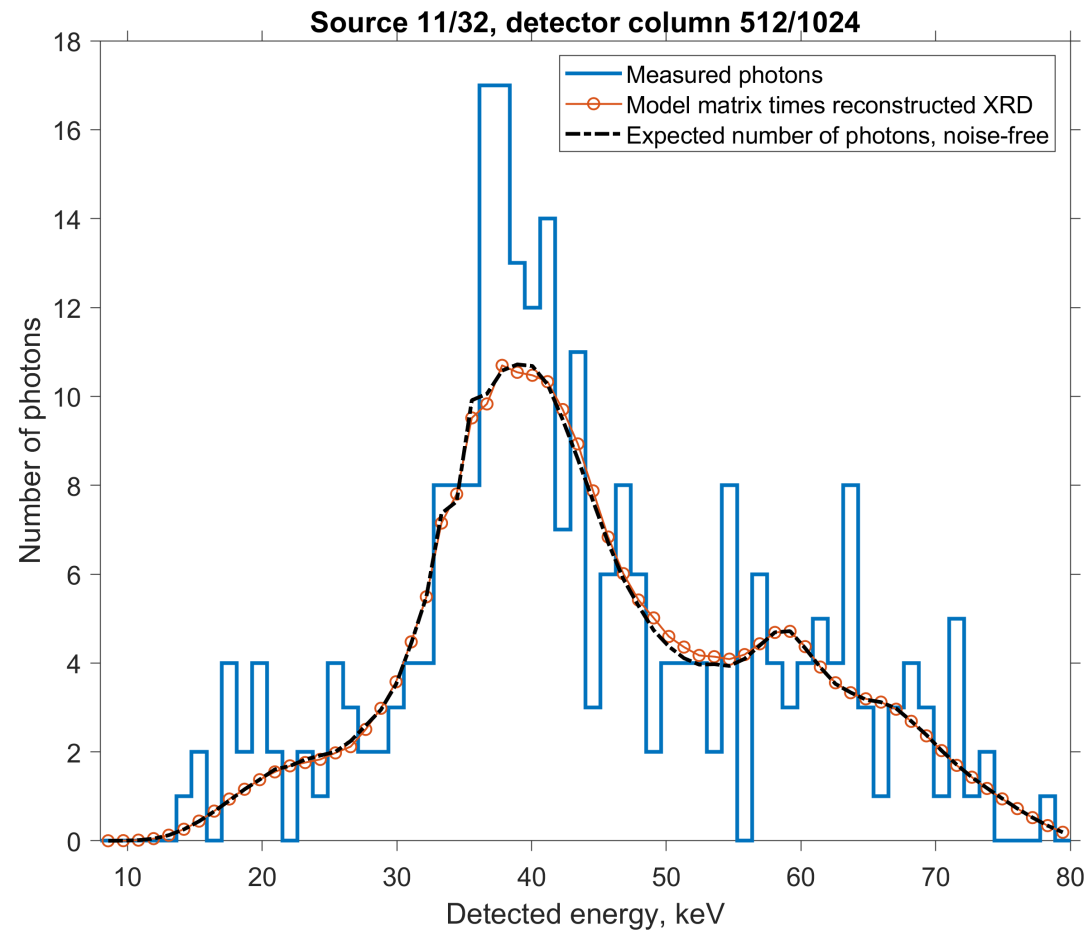

Fig. 10. One-dimensional slice of the noisy measurement from Fig. 9 For comparison, we show the number of photons $\mathbf{N}=\mathbb{A} \cdot \mathbf{F}$, computed using the reconstructed $\mathbf{F}$, as well as the noise-free ground truth calculation for the number of photons, Eq. (2). 


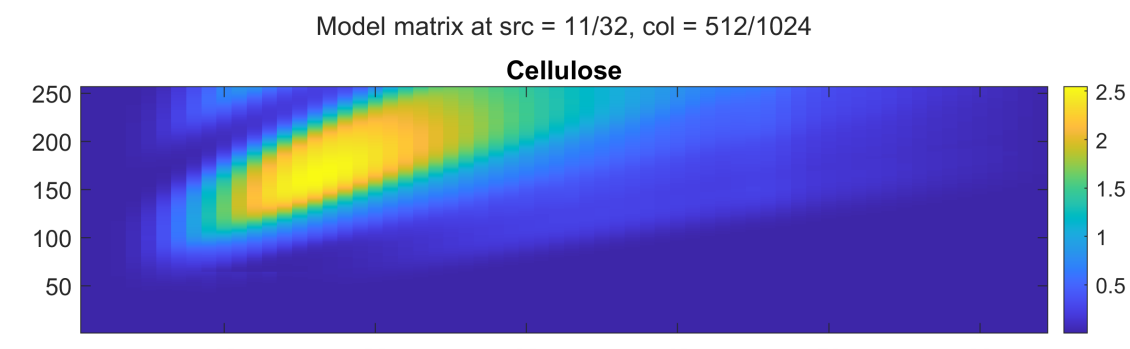

10

20

30

40

50

60

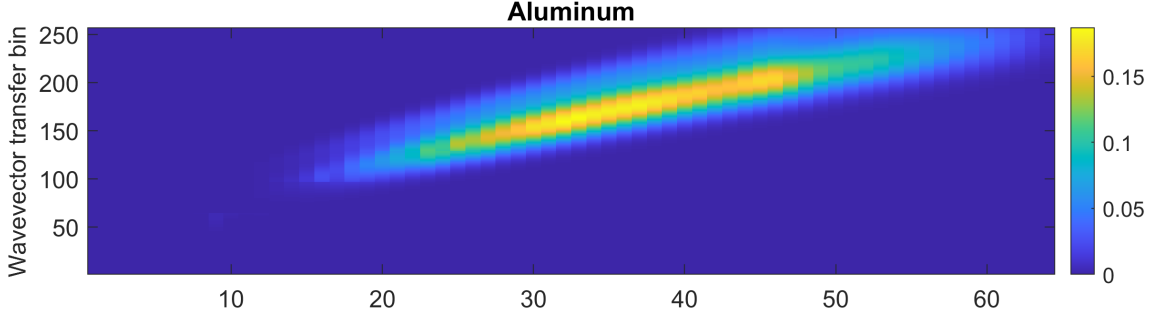

Ammonium nitrate

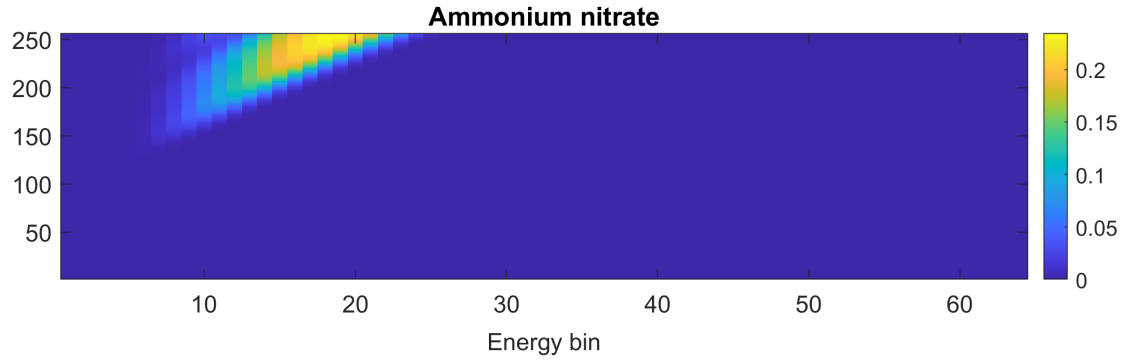

Fig. 11. One slice of the model matrix $\mathbb{A}$.

demonstrated results (see Fig. 12 may be insufficient. In a future study a number of techniques could be used to improve the accuracy and resolution of XRD inversion, including

- edge-preserving and detail-preserving regularization,

- enforcing sparsity in a suitable basis (e.g., in a wavelet basis),

- searching for diffraction patterns from a low-dimensional functional space (e.g., smooth background plus a few spikes with unknown locations and heights),

- deep learning, etc., see [33]-[36] and references therein.

The scan example that we have shown is fairly generic, and could be further optimized for more specific applications in domains other than security, for example healthcare or manufacturing quality control. Candidate areas include imaging defects in concrete [37] and metals [38], especially for quality control in 3D printing [39], as well as revealing the nanostructure of bones [40] and calcifications in soft tissues like breast [41]. The nanostructure of materials can be inferred by fitting a molecular model to the reconstructed diffraction pattern. For example, in a simple 1D case, Bragg's law says that a diffraction peak at $q=1 \AA^{-1}$ corresponds to a lattice of atoms with spacing $d=2 \pi / q=0.63 \mathrm{~nm}$.

In niche applications like those mentioned above, there are only a small number of diffracting materials, often known beforehand. In addition, the CT modality provides all the spatial information (locations, shapes of objects), as well as some information on material composition (density and average atomic number). In that case, there may be no need to run the costly and error-prone XRD inversion, Eq. [59]. It could be much faster and more robust to just run forward simulations using the matrix $\mathbb{A}$ and a list of known diffraction patterns stored in the column $\mathbf{F}$. We then only need to check which among the possible sets of materials gives rise to data closest to the measurement $\mathbf{N}$. Very often, the purpose of an $\mathrm{X}$-ray scan is to answer a simple yes/no question (threat vs benign, cancer vs healthy, etc.). In addition, the materials involved in a given application are often known beforehand. Even in the difficult case of airport security, there are no more than a few dozen kinds of explosives that an attacker is likely to carry in a suitcase. The number of commonly smuggled illegal drug types is even smaller, so if we could cost-effectively catch just a few of the most popular ones, that would aid law enforcement tremendously.

In conclusion, an XRD imaging add-on to existing CT scanners is feasible and is well-positioned to provide unique, materialspecific information, at a low cost of installing an extra detector or two, and developing suitable reconstruction software. 
Iteration $721 / 1000$
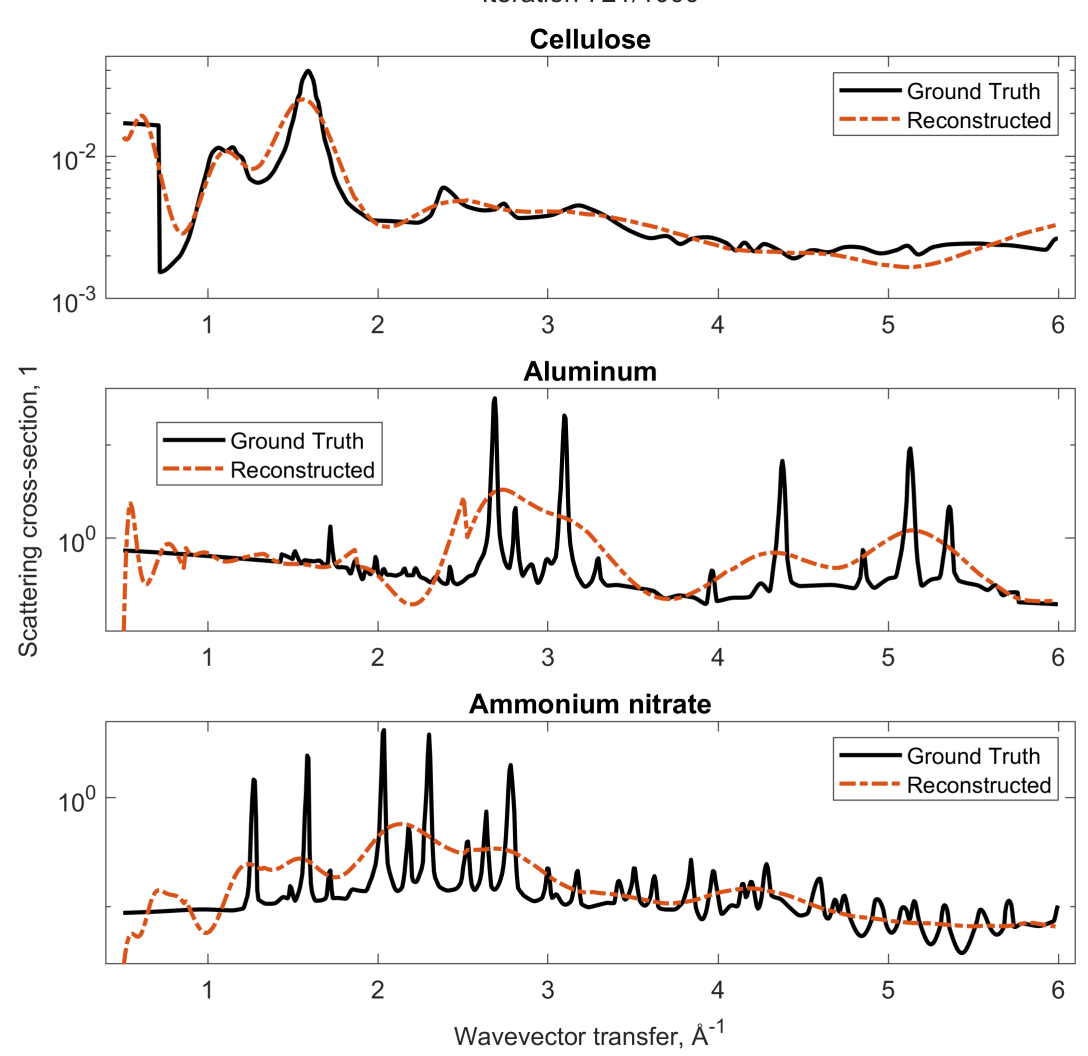

Fig. 12. Reconstructed vs ground-truth diffraction patterns on a logarithmic scale.

\section{ACKNOWLEDGMENT}

We acknowledge discussions on X-ray imaging technology with Anders Priest and Jacob Conn from Rapiscan Systems. We acknowledge useful correspondence with Theyencheri Narayanan from ESRF on SAXS and WAXS data calibration. A. Katsevich is a shareholder and chief technology officer of iTomography Corporation and, as such, may benefit financially as a result of the outcomes of the work reported in this publication.

\section{REFERENCES}

[1] J.-P. Schlomka, A. Harding, U. Van Stevendaal, M. Grass, and G. L. Harding, "Coherent scatter computed tomography: a novel medical imaging technique," in Medical Imaging 2003: Physics of Medical Imaging, vol. 5030. International Society for Optics and Photonics, 2003, pp. 256-265.

[2] R. W. Madden, J. Mahdavieh, R. C. Smith, and R. Subramanian, "An explosives detection system for airline security using coherent X-ray scattering technology," in Hard X-Ray, Gamma-Ray, and Neutron Detector Physics X, vol. 7079. International Society for Optics and Photonics, 2008 , p. 707915.

[3] G. Artioli, T. Cerulli, G. Cruciani, M. Dalconi, G. Ferrari, M. Parisatto, A. Rack, and R. Tucoulou, "X-ray diffraction microtomography (xrd-ct), a novel tool for non-invasive mapping of phase development in cement materials," Analytical and bioanalytical chemistry, vol. 397, no. 6, pp. 2131-2136, 2010

[4] J. A. Greenberg, M. N. Lakshmanan, D. J. Brady, and A. J. Kapadia, "Optimization of a coded aperture coherent scatter spectral imaging system for medical imaging," in Medical Imaging 2015: Physics of Medical Imaging, vol. 9412. International Society for Optics and Photonics, 2015, p. 94125E.

[5] K. Chen and D. A. Castañón, "A regularized iterative reconstruction algorithm for x-ray diffraction tomography," in Next-Generation Spectroscopic Technologies V, vol. 8374. International Society for Optics and Photonics, 2012, p. 83740D.

[6] — , "Architectures and algorithms for x-ray diffraction imaging," in Computational Imaging XII, vol. 9020. International Society for Optics and Photonics, 2014, p. 902006.

[7] B. Ghammraoui, A. Badal, and L. M. Popescu, "Maximum-likelihood estimation of scatter components algorithm for x-ray coherent scatter computed tomography of the breast," Physics in Medicine \& Biology, vol. 61, no. 8, p. 3164, 2016.

[8] R. Behling, Modern Diagnostic X-Ray Sources: Technology, Manufacturing, Reliability. CRC Press, 2015.

[9] D. O’Flynn, C. Reid, C. Christodoulou, M. Wilson, M. Veale, P. Seller, D. Hills, H. Desai, B. Wong, and R. Speller, "Explosive detection using pixellated x-ray diffraction (pixd)," Journal of Instrumentation, vol. 8, no. 03, p. P03007, 2013.

[10] G. Harding, "X-ray scatter tomography for explosives detection," Radiation Physics and Chemistry, vol. 71, no. 3-4, pp. 869-881, 2004.

[11] G. Harding and A. Harding, "X-ray diffraction imaging for explosives detection," in Counterterrorist detection techniques of explosives. Elsevier, 2007, pp. 199-235.

[12] J.-P. Schlomka, J. Delfs, H. Barschdorf, A. Thran, and U. van Stevendaal, "Experimental feasibility study of energy-resolved fan-beam coherent scatter computed tomography," in Developments in X-Ray Tomography IV, vol. 5535. International Society for Optics and Photonics, 2004, pp. 410-423.

[13] J.-P. Schlomka, S. M. Schneider, and G. L. Harding, "Novel concept for coherent scatter x-ray computed tomography in medical applications," in Penetrating Radiation Systems and Applications II, vol. 4142. International Society for Optics and Photonics, 2000, pp. 218-224.

[14] S. M. Schneider, J.-P. Schlomka, and G. L. Harding, "Coherent-scatter computed tomography applying a fan-beam geometry," in Medical Imaging 2001: Physics of Medical Imaging, vol. 4320. International Society for Optics and Photonics, 2001, pp. 754-763. 
Iteration $721 / 1000$
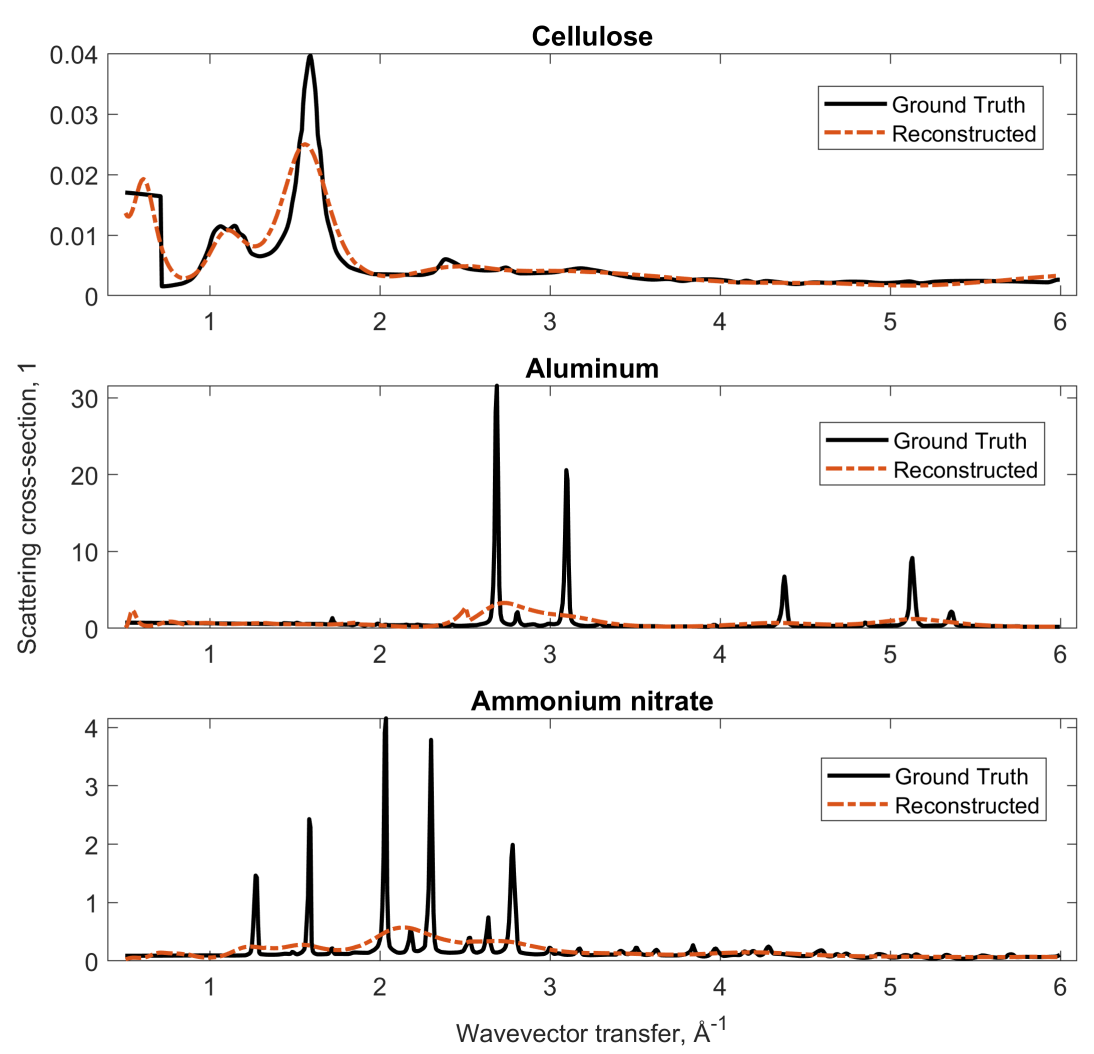

Fig. 13. Reconstructed vs ground-truth diffraction patterns on a linear scale.

[15] J.-P. Schlomka and M. Gra $\beta$, "Computed tomography device and method with three-dimensional backprojection,” Aug. 26 2008, uS Patent 7,418,073.

[16] J. Delfs and J.-P. Schlomka, "Energy-dispersive coherent scatter computed tomography," Applied physics letters, vol. 88, no. 24, p. $243506,2006$.

[17] A. D. Holmgren, I. Odinaka, J. A. Greenberg, and D. J. Brady, "Multi-view coded aperture coherent scatter tomography," in Anomaly Detection and Imaging with X-Rays (ADIX), vol. 9847. International Society for Optics and Photonics, 2016, p. 98470A.

[18] Z. Zhu, A. Katsevich, A. J. Kapadia, J. A. Greenberg, and S. Pang, "X-ray diffraction tomography with limited projection information," Scientific reports, vol. 8, no. 1, pp. 1-6, 2018.

[19] J.-P. Schlomka, "Coherent-scatter computer tomograph," Sep. 15 2009, uS Patent 7,590,215.

[20] U. Van Stevendaal, J.-P. Schlomka, A. Harding, and M. Grass, "A reconstruction algorithm for coherent scatter computed tomography based on filtered back-projection," Medical Physics, vol. 30, no. 9, pp. 2465-2474, 2003.

[21] J. Als-Nielsen and D. McMorrow, Elements of modern X-ray physics. John Wiley \& Sons, 2011.

[22] M. Król, P. Snopinski, B. Tomiczek, T. Tanski, W. Pakiela, and W. Sitek, "Structure and properties of an al alloy in as-cast state and after laser treatment," Proceedings of the Estonian Academy of Sciences, vol. 65, no. 2, p. 107, 2016.

[23] A. Bota, "Development of powder diffraction apparatus for small-angle x-ray scattering measurements," Journal of Applied Crystallography, vol. 46, no. 2, pp. 573-576, 2013.

[24] J. Ilavsky, F. Zhang, R. N. Andrews, I. Kuzmenko, P. R. Jemian, L. E. Levine, and A. J. Allen, "Development of combined microstructure and structure characterization facility for in situ and operando studies at the advanced photon source," Journal of applied crystallography, vol. 51, no. 3, pp. 867-882, 2018.

[25] P. Panine, E. Di Cola, M. Sztucki, and T. Narayanan, "Early stages of polymer melt crystallization,” Polymer, vol. 49, no. 3, pp. 676-680, 2008.

[26] T. Narayanan, M. Sztucki, P. Van Vaerenbergh, J. Léonardon, J. Gorini, L. Claustre, F. Sever, J. Morse, and P. Boesecke, "A multipurpose instrument for time-resolved ultra-small-angle and coherent x-ray scattering," Journal of applied crystallography, vol. 51, no. 6, pp. 1511-1524, 2018.

[27] J. H. Hubbell, W. J. Veigele, E. Briggs, R. Brown, D. Cromer, and d. R. Howerton, "Atomic form factors, incoherent scattering functions, and photon scattering cross sections," Journal of physical and chemical reference data, vol. 4, no. 3, pp. 471-538, 1975.

[28] J. Schlomka, E. Roessl, R. Dorscheid, S. Dill, G. Martens, T. Istel, C. Bäumer, C. Herrmann, R. Steadman, G. Zeitler et al., "Experimental feasibility of multi-energy photon-counting k-edge imaging in pre-clinical computed tomography," Physics in Medicine \& Biology, vol. 53, no. 15, p. 4031, 2008.

[29] K. Taguchi, C. Polster, O. Lee, K. Stierstorfer, and S. Kappler, "Spatio-energetic cross talk in photon counting detectors: Detector model and correlated poisson data generator," Medical physics, vol. 43, no. 12, pp. 6386-6404, 2016.

[30] A. Thran, U. van Stevendaal, and J.-P. Schlomka, "Multiple scatter correction in coherent scatter computed tomography," in Penetrating Radiation Systems and Applications VII, vol. 5923. International Society for Optics and Photonics, 2005, p. 59230P.

[31] X. Ju, M. Bowden, E. E. Brown, and X. Zhang, "An improved x-ray diffraction method for cellulose crystallinity measurement," Carbohydrate polymers, vol. 123 , pp. 476-481, 2015.

[32] S. Welzmiller and H. Pilliere, "Explosives in forensic science using benchtop arl equinox 100 x-ray diffractometer," https://assets.thermofisher.com/ TFS-Assets/MSD/Application-Notes/XRD-AN41129-explosive-material-forensic-study-arl-equinox-100.pdf 2021, accessed: 2021-7-6.

[33] A. Matakos, S. Ramani, and J. A. Fessler, “Accelerated Edge-Preserving Image Restoration Without Boundary Artifacts," IEEE Transactions on Image Processing, vol. 22, pp. 2019-2029, 2013.

[34] U. S. Kamilov, I. N. Papadopoulos, M. H. Shoreh et al., "Optical Tomographic Image Reconstruction Based on Beam Propagation and Sparse Regularization," IEEE Transactions on Computational Imaging, vol. 2, pp. 59-70, 2016. 
[35] J. Liu, Y. Sun, C. Eldeniz et al., "RARE: Image Reconstruction Using Deep Priors Learned Without Groundtruth," IEEE Journal of Selected Topics in Signal Processing, vol. 14, pp. 1088-1099, 2020.

[36] S. Ravishankar, J. C. Ye, and J. A. Fessler, "Image Reconstruction: From Sparsity to Data-Adaptive Methods and Machine Learning," Proceedings of the IEEE, vol. 108, pp. 86-109, 2020.

[37] M. Paul, "Quality control of autoclaved aerated concrete by means of x-ray diffraction," ce/papers, vol. 2, no. 4, pp. 111-116, 2018.

[38] M. J. Cherukara, R. Pokharel, T. S. O’Leary, J. K. Baldwin, E. Maxey, W. Cha, J. Maser, R. J. Harder, S. J. Fensin, and R. L. Sandberg, "Threedimensional x-ray diffraction imaging of dislocations in polycrystalline metals under tensile loading," Nature communications, vol. 9, no. 1, pp. 1-6, 2018.

[39] C. Zhao, K. Fezzaa, R. W. Cunningham, H. Wen, F. De Carlo, L. Chen, A. D. Rollett, and T. Sun, "Real-time monitoring of laser powder bed fusion process using high-speed x-ray imaging and diffraction," Scientific reports, vol. 7, no. 1, pp. 1-11, 2017.

[40] S. Tadano and B. Giri, "X-ray diffraction as a promising tool to characterize bone nanocomposites," Science and technology of advanced materials, 2012.

[41] R. Scott, N. Stone, C. Kendall, K. Geraki, and K. Rogers, "Relationships between pathology and crystal structure in breast calcifications: an in situ x-ray diffraction study in histological sections," NPJ Breast Cancer, vol. 2, no. 1, pp. 1-6, 2016.

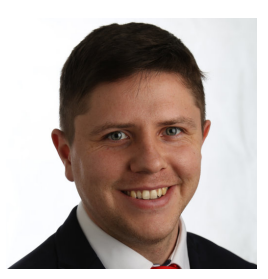

Airidas Korolkovas was born in Kaunas, Lithuania, in 1989. He has received the B.S. degree in Industrial Engineering from Politecnico di Torino, Italy, in 2011, the M.S. degree in Nanoscience and Nanotechnology from KU Leuven, Belgium, in 2013, and the Ph.D. degree in Nanophysics from Grenoble Alpes University, France, in 2016. From 2013 to 2017 he was a graduate researcher with the Institut Laue-Langevin, where he used neutron scattering, rheology, and computer simulations to study non-linear polymer behavior under confinement and strong flow. From 2017 to 2018 he was a postdoctoral researcher with Uppsala University, Sweden, where he developed GPU-accelerated simulations that run up to 100-times faster than published in previous literature. Since 2019, he has been working as an X-ray physicist and imaging scientist with iTomography Corporation, Houston, TX, USA. His current research interests include tomographic and coherent imaging with neutron, X-ray, optical, and other probes, combined with machine learning and deep neutral networks, to serve the needs of healthcare, petroleum, security, and other industries.

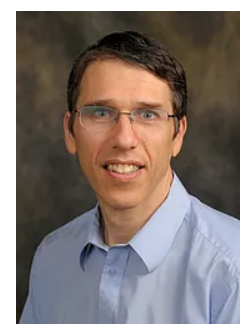

Alexander Katsevich received the Ph.D. degree in Mathematics from Kansas State University, Manhattan, KS, in 1994. Since 1996, he has been a Professor with the Mathematics Department at the University of Central Florida. In 2011, he co-founded iTomography and has been the company CTO since then. He is the author of one book, more than 90 papers, and more than 25 inventions. His research interests include development of image reconstruction algorithms in tomography and their mathematical analysis. He is on the Editorial Board of the journal Inverse Problems. Dr. Katsevich secured multiple research grants from the National Science Foundation as a PI. He received Alexander von Humboldt Research Fellowship in 2005, and the Best paper in tomography Award at the IXth International Conference on Fully 3D Image Reconstruction in Radiology and Nuclear Medicine (Lindau, Germany, July 2007). Dr. Katsevich is a recipient of the 2016 Marcus Wallenberg Prize (Sweden) for the development of an ultra-fast computed tomography scanner for whole tree logs.

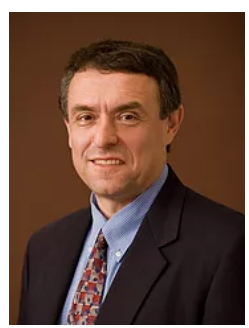

Michael Frenkel is President \& CEO of iTomography, which he co-founded in Houston in 2011, with the mission to develop cutting-edge CT imaging solutions for medical and industrial applications. In addition to leading the company's business and technical development with major manufacturers and end-users of CT equipment, he has managed clinical studies and collaborations at the Texas Medical Center in Houston and with the National Institutes of Health. Previously, in 20072010, he was the Vice President of Geology and Geophysics at EMGS Americas in Houston, where he managed marine Controlled-Source Electromagnetic (CSEM) exploration projects for major oil companies. While working at the Baker Hughes Houston Technology Center from 1992 to 2007, he led R\&D of new EM well-logging data acquisition tools and inversionbased formation imaging methods for hydrocarbon exploration \& production. His research interests include data processing, fast media imaging, and development of novel tomographic acquisition systems. He has published a monograph, a number of papers, and was awarded 17 patents in Electromagnetic and CT imaging. Dr. Frenkel holds a Ph.D. in Physics and Mathematics from the Institute of Terrestrial Magnetism, Ionosphere and Radio Wave Propagation (Moscow, 1984).

PLACE

PHOTO HERE
William Thompson received his PhD degree from the University of Manchester, UK in 2011. This covered aspects of reconstruction algorithms and image quality for a novel geometry, switched source x-ray CT scanner. Since then, he has continued to work on image reconstruction problems in both academic and industrial settings, with a focus on real-time computing and high-performance GPU implementations. He joined Rapiscan Systems in early 2018, where he oversaw development of a new real-time image processing and reconstruction pipeline for the company's next generation CT scanners. In his current role, he leads an international team of algorithm developers working on a range of image processing and detection problems across a breadth of imaging modalities and applications. 


\begin{tabular}{|c|}
\hline \\
PLACE \\
PHOTO \\
HERE \\
\end{tabular}

Edward Morton received his PhD degree from the University of London, UK in 1988. He is a Fellow of the Royal Academy of Engineering, and is the lead author of numerous scientific papers and patents in x-ray imaging and related fields. He is the inventor of the Real Time Tomography (RTT) system, a revolutionary type of tomographic x-ray scanner with no moving parts. He formed the startup company CXR in the United Kingdom to develop the RTT concept into a commercial product. As a successful startup, CXR was acquired by Rapiscan Systems in 2007, where the RTT system is now a successful product being deployed in airports around the world. He continues to serve as Chief Technology Officer for Rapiscan Systems, where he oversees development of the next generation of security imaging technology. 